This item was submitted to Loughborough's Research Repository by the author.

Items in Figshare are protected by copyright, with all rights reserved, unless otherwise indicated.

\title{
On integrability in Grassmann geometries: integrable systems associated with fourfolds in $\operatorname{Gr}(3,5)$
}

\section{PLEASE CITE THE PUBLISHED VERSION}

https://doi.org/10.1112/plms.12114

\section{PUBLISHER}

Wiley (@ London Mathematical Society)

\section{VERSION}

AM (Accepted Manuscript)

\section{PUBLISHER STATEMENT}

This work is made available according to the conditions of the Creative Commons Attribution-NonCommercial 4.0 International (CC BY-NC 4.0) licence. Full details of this licence are available at: https://creativecommons.org/licenses/by-nc/4.0/

\section{LICENCE}

CC BY-NC 4.0

\section{REPOSITORY RECORD}

Doubrov, B., Evgeny Ferapontov, B. Kruglikov, and Vladimir Novikov. 2018. "On Integrability in Grassmann Geometries: Integrable Systems Associated with Fourfolds in $\operatorname{Gr}(3,5)$ ". Loughborough University. https://hdl.handle.net/2134/27833. 


\title{
On integrability in Grassmann geometries: integrable systems associated with fourfolds in $\mathbf{G r}(3,5)$
}

B. Doubrov ${ }^{1}$, E.V. Ferapontov ${ }^{2}$, B. Kruglikov ${ }^{3}$, V.S. Novikov ${ }^{2}$

\author{
${ }^{1}$ Department of Mathematical Physics \\ Faculty of Applied Mathematics \\ Belarussian State University \\ Nezavisimosti av. 4, 220030 Minsk, Belarus \\ ${ }^{2}$ Department of Mathematical Sciences \\ Loughborough University \\ Loughborough, Leicestershire LE11 3TU \\ United Kingdom \\ ${ }^{3}$ Department of Mathematics and Statistics \\ UiT the Arctic University of Norway \\ Tromsø 90-37, Norway

$$
\text { e-mails: }
$$ \\ doubrov@islc.org \\ E.V.Ferapontov@lboro.ac.uk \\ boris.kruglikov@uit.no \\ V.Novikov@lboro.ac.uk
}




\begin{abstract}
Let $\mathbf{G r}(d, n)$ be the Grassmannian of $d$-dimensional linear subspaces of an $n$-dimensional vector space $V^{n}$. A submanifold $X \subset \mathbf{G r}(d, n)$ gives rise to a differential system $\Sigma(X)$ that governs $d$-dimensional submanifolds of $V^{n}$ whose Gaussian image is contained in $X$. Systems of the form $\Sigma(X)$ appear in numerous applications in continuum mechanics, theory of integrable systems, general relativity and differential geometry. They include such wellknown examples as the dispersionless Kadomtsev-Petviashvili equation, the Boyer-Finley equation, Plebańsky's heavenly equations, and so on.

In this paper we concentrate on the particularly interesting case of this construction where $X$ is a fourfold in $\mathbf{G r}(3,5)$. Our main goal is to investigate differential-geometric and integrability aspects of the corresponding systems $\Sigma(X)$. We demonstrate the equivalence of several approaches to dispersionless integrability such as

- the method of hydrodynamic reductions,

- the method of dispersionless Lax pairs,

- integrability on solutions, based on the requirement that the characteristic variety of system $\Sigma(X)$ defines an Einstein-Weyl geometry on every solution,

- integrability on equation, meaning integrability (in twistor-theoretic sense) of the canonical $G L(2, \mathbb{R})$ structure induced on a fourfold $X \subset \mathbf{G r}(3,5)$.

All these seemingly different approaches lead to one and the same class of integrable systems $\Sigma(X)$. We prove that the moduli space of such systems is 6 -dimensional. We give a complete description of linearisable systems (the corresponding fourfold $X$ is a linear section of $\operatorname{Gr}(3,5)$ ) and linearly degenerate systems (the corresponding fourfold $X$ is the image of a quadratic map $\mathbb{P}^{4} \rightarrow \mathbf{G r}(3,5)$ ). The fourfolds corresponding to 'generic' integrable systems are not algebraic, and can be parametrised by generalised hypergeometric functions.
\end{abstract}

MSC: 37K10, 37K25, 53A30, 53A40, 53B15, 53B25, 53B50, 53Z05.

Keywords: Dispersionless Integrable System, Dispersionless Lax Pair, Hydrodynamic Reduction, Einstein-Weyl Geometry, Submanifold of the Grassmannian, GL(2, R) Geometry. 


\section{Contents}

1 Introduction $\quad 4$

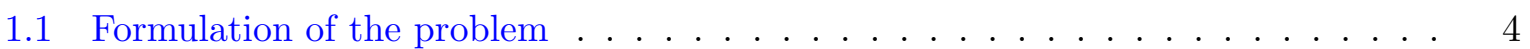

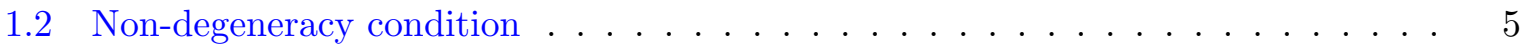

1.3 Einstein-Weyl geometry in $3 \mathrm{D} \ldots \ldots \ldots \ldots \ldots \ldots$

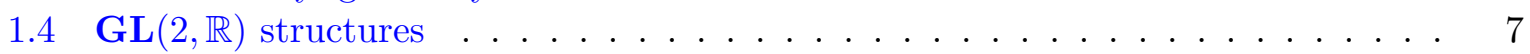

1.5 The method of hydrodynamic reductions . . . . . . . . . . . 7

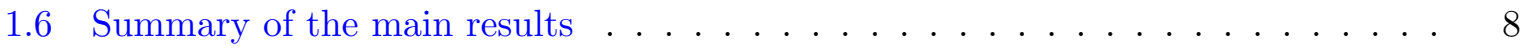

2 Integrability conditions and Lax pairs $\quad 9$

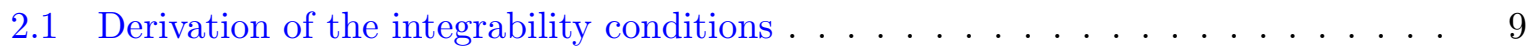

2.2 Dispersionless Lax pairs . . . . . . . . . . . . . . . . . . . . . 12

2.3 Lax pairs and null totally geodesic surfaces . . . . . . . . . . . 13

$3 \mathrm{SL}(5)$ as the equivalence group: geometry of $\operatorname{Gr}(3,5) \quad 14$

4 Examples and classification results $\quad 17$

4.1 Linearisable systems . . . . . . . . . . . . . . . . . . . . . . 17

4.2 Linearly degenerate systems . . . . . . . . . . . . . . . . . . . 19

4.3 Further examples in terms of modular forms and theta functions . . . . . . . . 25

4.4 Integrable systems in terms of generalised hypergeometric functions $\ldots \ldots$. . . 26

5 Geometric aspects of integrability $\quad \mathbf{2 7}$

$5.1 \mathbf{G L}(2, \mathbb{R})$ structure on a fourfold $X \subset \mathbf{G r}(3,5) \ldots \ldots \ldots \ldots . \ldots 27$

5.2 Geometric interpretation of the integrability conditions . . . . . . . . . . 28

5.3 Canonical conformal almost symplectic structure . . . . . . . . . . . . 30

5.4 Integrability conditions via differential invariants . . . . . . . . . . . . . 31

6 Concluding remarks $\quad 33$ 


\section{Introduction}

\subsection{Formulation of the problem}

In the most general setting the problem that we address in this paper can be described as follows. Let $\operatorname{Gr}(d, n)$ be the Grassmannian of $d$-dimensional linear subspaces of an $n$-dimensional vector space $V^{n}$. A submanifold $X \subset \mathbf{G r}(d, n)$ gives rise to the differential system $\Sigma(X)$ that governs $d$-dimensional submanifolds of $V^{n}$ whose Gaussian image is contained in $X$ (we recall that the Gaussian image of a submanifold is the collection of its tangent spaces translated to the origin). In this sense we are in the context of Grassmann geometries as discussed in [36]. Since $d$-dimensional submanifolds of $V^{n}$ are (locally) parametrised by $n-d$ functions of $d$ variables, we will assume that the codimension of $X$ in $\mathbf{G r}(d, n)$ also equals $n-d$ : in this case $\Sigma(X)$ will be a determined system of $n-d$ first-order PDEs for $n-d$ unknown functions of $d$ independent variables. Systems of the form $\Sigma(X)$ appear in a wide range of applications in differential geometry (in particular, calibrated geometries [36]), general relativity (Einstein-Weyl structures [19], heavenly-type equations [50]), and the theory of integrable systems (Bäcklund transformations in higher dimensions, dispersionless Lax pairs [62], dispersionless limits of multi-dimensional soliton equations). Particularly interesting examples are provided by multidimensional $(d \geq 3)$ integrable systems $\Sigma(X)$ : such systems possess an infinity of multi-phase solutions that can be considered as analogues of multi-gap solutions of integrable soliton equations. It was observed in [24] that the requirement of the existence of such solutions constitutes an efficient criterion (known as the method of hydrodynamic reductions: see Sect. 1.5 for a brief description of the method), that allows one to classify integrable systems of the form $\Sigma(X)$. In this paper we concentrate on the following key questions:

- What is the dimension of the moduli space of integrable systems $\Sigma(X)$ ? What are the most interesting examples?

- How does integrability of a system $\Sigma(X)$ translate into differential geometry of the corresponding submanifold $X \subset \mathbf{G r}(d, n)$ ?

In the case $n=d+1$ the system $\Sigma(X)$ consists of a single first-order PDE for a scalar function of $d$ independent variables which can be solved by the method of characteristics via reduction to ODEs. Thus, we can assume $n \geq d+2$. The case $d=1$ also corresponds to an ODE system. The case $d=2, n$ arbitrary, leads to another familiar class of systems: introducing in $V^{n}$ coordinates $t, x, u^{i}, i=1, \ldots, n-2$, and parametrising two-dimensional submanifolds of $V^{n}$ in the form $u^{i}=u^{i}(t, x)$, we can represent the corresponding system $\Sigma(X)$ as

$$
F^{1}\left(u_{t}^{i}, u_{x}^{i}\right)=0, \quad \ldots, \quad F^{n-2}\left(u_{t}^{i}, u_{x}^{i}\right)=0 .
$$

Solving these equations for $u_{t}^{i}$ in the form $u_{t}^{i}=f^{i}\left(\mathbf{u}_{x}\right)$, here $\mathbf{u}=\left(u^{1}, \ldots, u^{n-2}\right)$, differentiating by $x$ and setting $\mathbf{u}_{x}=\mathbf{v}$, we obtain a system of conservation laws,

$$
v_{t}^{i}=\partial_{x} f^{i}(\mathbf{v}) .
$$

Differential-geometric and integrability aspects of such systems were extensively studied in [58, $54,18,2]$, see also references therein. Thus, in what follows we assume $n \geq d+2$ and $d \geq 3$.

To be more specific we will concentrate on the particularly interesting case of this problem (which however contains all essential challenges of the general theory) where our results are 
fairly complete, namely the case of fourfolds $X \subset \mathbf{G r}(3,5)(n=5, d=3)$. Introducing in $V^{5}$ coordinates $x^{1}, x^{2}, x^{3}, u, v$, one can parametrise three-dimensional submanifolds of $V^{5}$ in the form $u=u\left(x^{1}, x^{2}, x^{3}\right), v=v\left(x^{1}, x^{2}, x^{3}\right)$. Their tangent spaces are given by $d u=u_{i} d x^{i}, d v=v_{i} d x^{i}$ where $u_{i}, v_{i}$ can be viewed as local coordinates on $\mathbf{G r}(3,5)$. The corresponding system $\Sigma(X)$ reduces to a pair of first-order PDEs for $u$ and $v$,

$$
F\left(u_{1}, u_{2}, u_{3}, v_{1}, v_{2}, v_{3}\right)=0, \quad G\left(u_{1}, u_{2}, u_{3}, v_{1}, v_{2}, v_{3}\right)=0
$$

$u_{i}=\partial u / \partial x^{i}, v_{i}=\partial v / \partial x^{i}$. Equations (1) specify a fourfold $X \subset \mathbf{G r}(3,5)$. The class of systems (1) is invariant under the equivalence group $\mathbf{S L}(5)$ that acts by linear transformations on the combined set of variables $x^{1}, x^{2}, x^{3}, u, v$. Since this action preserves the integrability, all our classification results will be formulated modulo SL(5)-equivalence. Necessary details on the equivalence group are provided in Sect. 3.

There exists a whole variety of important examples that fall into class (1). One of them appears in the context of the dispersionless Kadomtsev-Petviashvili (dKP) equation, $u_{x t}-u_{x} u_{x x}-$ $u_{y y}=0$, one of the most well-studied dispersionless integrable PDEs arising in nonlinear acoustics [60] and the theory of Einstein-Weyl structures [19]. Its dispersionless Lax pair [62] consists of two first-order relations of type (1),

$$
v_{y}-\frac{1}{2} v_{x}^{2}-u_{x}=0, \quad v_{t}-\frac{1}{3} v_{x}^{3}-v_{x} u_{x}-u_{y}=0,
$$

here $\left(x^{1}, x^{2}, x^{3}\right)=(x, y, t)$. The dKP equation results from (2) on elimination of $v$, that is, via the compatibility condition $v_{y t}=v_{t y}$. Similarly, the elimination of $u$ leads to the modified dKP (mdKP) equation, $v_{x t}-\left(v_{y}-\frac{1}{2} v_{x}^{2}\right) v_{x x}-v_{y y}=0$. Thus, relations (2) provide Bäcklund-type transformation connecting dKP and mdKP equations. We refer to Sect. 4 for further examples and classification results.

\subsection{Non-degeneracy condition}

In what follows we assume that system (1) is non-degenerate in the following sense. Let us consider the corresponding linearised system,

$$
\left(\begin{array}{ll}
F_{u_{1}} & F_{v_{1}} \\
G_{u_{1}} & G_{v_{1}}
\end{array}\right)\left(\begin{array}{l}
\mathcal{U} \\
\mathcal{V}
\end{array}\right)_{x^{1}}+\left(\begin{array}{ll}
F_{u_{2}} & F_{v_{2}} \\
G_{u_{2}} & G_{v_{2}}
\end{array}\right)\left(\begin{array}{l}
\mathcal{U} \\
\mathcal{V}
\end{array}\right)_{x^{2}}+\left(\begin{array}{ll}
F_{u_{3}} & F_{v_{3}} \\
G_{u_{3}} & G_{v_{3}}
\end{array}\right)\left(\begin{array}{l}
\mathcal{U} \\
\mathcal{V}
\end{array}\right)_{x^{3}}=0
$$

obtained by setting $u \rightarrow u+\epsilon \mathcal{U}, v \rightarrow v+\epsilon \mathcal{V}$, expanding $F$ and $G$ in Taylor series and keeping terms of the order $\epsilon$. Non-degeneracy means that the dispersion relation (characteristic variety),

$$
\operatorname{det}\left[\lambda^{1}\left(\begin{array}{cc}
F_{u_{1}} & F_{v_{1}} \\
G_{u_{1}} & G_{v_{1}}
\end{array}\right)+\lambda^{2}\left(\begin{array}{cc}
F_{u_{2}} & F_{v_{2}} \\
G_{u_{2}} & G_{v_{2}}
\end{array}\right)+\lambda^{3}\left(\begin{array}{cc}
F_{u_{3}} & F_{v_{3}} \\
G_{u_{3}} & G_{v_{3}}
\end{array}\right)\right]=0,
$$

defines an irreducible conic in $\mathbb{P}^{2}$ with homogeneous coordinates $\left(\lambda^{1}: \lambda^{2}: \lambda^{3}\right)$. Explicitly, the dispersion relation can be represented in the form $\left(\lambda^{1}, \lambda^{2}, \lambda^{3}\right) g^{\sharp}\left(\lambda^{1}, \lambda^{2}, \lambda^{3}\right)^{t}=0$ where $g^{\sharp}$ is the $3 \times 3$ symmetric matrix,

$$
g^{\sharp}=g^{i j}=\frac{1}{2}\left(F_{u^{i}} G_{v^{j}}+F_{u^{j}} G_{v^{i}}-F_{v^{i}} G_{u^{j}}-F_{v^{j}} G_{u^{i}}\right) .
$$

It gives rise to the conformal structure $g=g_{i j} d x^{i} d x^{j}$ (here $g_{i j}$ is the inverse of $g^{i j}$ ). Note that non-degeneracy is equivalent to $\operatorname{det} g \neq 0$ : this is the case for all known systems of physi$\mathrm{cal} /$ geometric relevance. It turns out that the signature of $g$ is always Lorentzian, and thus our PDE system is hyperbolic. 
Geometric aspects of conformal structures defined by the characteristic variety will play a key role in our characterisation of integrable systems: we will see that solutions to integrable equations carry integrable background geometry [11]. In 3D, this is the Einstein-Weyl geometry.

\subsection{Einstein-Weyl geometry in 3D}

Recall that an Einstein-Weyl geometry consists of a symmetric connection $\mathbb{D}$ and a conformal structure $g$ such that:

(a) connection $\mathbb{D}$ preserves the conformal class of $g: \mathbb{D}[g]=0$,

(b) trace-free part of the symmetrized Ricci tensor of $\mathbb{D}$ vanishes.

In coordinates, this gives

$$
\mathbb{D}_{k} g_{i j}=\omega_{k} g_{i j}, \quad R_{(i j)}=\Lambda g_{i j},
$$

where $\omega=\omega_{k} d x^{k}$ is a covector, $R_{(i j)}$ is the symmetrized Ricci tensor of $\mathbb{D}$, and $\Lambda$ is some function [12]. Note that it is sufficient to specify $g$ and $\omega$ only, then the first set of equations uniquely determines $\mathbb{D}$. The integrability of Einstein-Weyl equations (3) by twistor-theoretic methods was established by Hitchin [37]. It was shown in [23] that generic Einstein-Weyl structures are governed by the Manakov-Santini system introduced in [40] as a two-component integrable generalisation of the dKP equation. We will prove that for integrable systems (1) the conformal structure $g$ defined by the characteristic variety must be Einstein-Weyl on every solution, with the covector $\omega$ expressed in terms of $g$ by the universal formula

$$
\omega_{k}=2 g_{k j} \mathcal{D}_{x^{s}}\left(g^{j s}\right)+\mathcal{D}_{x^{k}}\left(\ln \operatorname{det} g_{i j}\right),
$$

where $\mathcal{D}_{x^{k}}$ denotes total derivative with respect to the independent variable $x^{k}$ (note that $g$ depends on first-order jets of the solution $u, v$ ). Formula (4) appeared in [28] in geometric approach to the dispersionless integrability in 3D. It is invariant under the gauge transformation $g \rightarrow \lambda g, \omega \rightarrow \omega+d \ln \lambda$, the property characteristic of Einstein-Weyl geometry. According to the result of Cartan [12], the Einstein-Weyl property of a triple $(\mathbb{D}, g, \omega)$ is equivalent to the existence of a two-parameter family of surfaces that are null with respect to the conformal structure $g$ (that is, tangential to the null cones of $g$ ), and totally geodesic in the Weyl connection $\mathbb{D}$. In the context of integrable systems (1), such surfaces are provided by the corresponding dispersionless Lax pairs: these consist of $\lambda$-dependent vector fields $X, Y$ that are required to commute modulo (1), identically in the 'spectral parameter' $\lambda$. For systems (1), the existence of such Lax pairs is equivalent to the integrability by the method of hydrodynamic reductions, see Sect. 2.2. Taking integral surfaces of the distribution spanned by $X, Y$ in the extended four-space with coordinates $x, y, t, \lambda$, and projecting them down to the space of the independent variables $x, y, t$, we obtain the required two-parameter family of null totally geodesic surfaces. Let us mention that relations of dispersionless integrable systems in 3D to Einstein-Weyl geometry have been discussed previously in [59, 11, 20, 28], see also references therein.

Example 1. Let us consider the system

$$
u_{t}-\frac{1}{2} u_{x}^{2}-v_{y}=0, \quad v_{x}-u_{y}=0,
$$

which reduces to the dKP equation, $u_{x t}-u_{x} u_{x x}-u_{y y}=0$, on elimination on $v$. Its characteristic variety defines the conformal structure $g=4 d x d t-d y^{2}+4 u_{x} d t^{2}$. Introducing the covector $\omega=$ $-4 u_{x x} d t$ by formula (4), one can verify that the pair $g, \omega$ satisfies the Einstein-Weyl equations if 
$u$ solves the dKP equation. This Einstein-Weyl structure was obtained previously in [19]. The corresponding Lax pair has the form

$$
X=\partial_{y}-\lambda \partial_{x}+u_{x x} \partial_{\lambda}, \quad Y=\partial_{t}-\left(\lambda^{2}+u_{x}\right) \partial_{x}+\left(u_{x x} \lambda+u_{x y}\right) \partial_{\lambda}
$$

one can verify that these vector fields commute modulo the above system. Projecting integral surfaces of the distribution spanned by $X, Y$ from the extended space of variables $x, y, t, \lambda$ to the space of independent variables $x, y, t$, one obtains a two-parameter family of null totally geodesic surfaces of the corresponding Einstein-Weyl structure.

\section{$1.4 \mathrm{GL}(2, \mathbb{R})$ structures}

The tangent bundle to the Grassmannian $\mathbf{G r}(3,5)$ carries canonical generalised conformal structure defined by the family of Segre cones $d u_{i} d v_{j}-d u_{j} d v_{i}=0$. Thus, each projectivised tangent space PTGr $(3,5)$ contains a Segre variety, an algebraic threefold of degree three. Given a nondegenerate fourfold $X \subset \mathbf{G r}(3,5)$ (a fourfold is said to be non-degenerate if it gives rise to a non-degenerate system (1)), the intersection of its tangent space $\mathrm{T} X$ with the Segre cone is a two-dimensional rational cone of degree three; its projectivisation is a rational normal curve of degree three (twisted cubic). Thus, $\mathbb{P T X}$ is supplied with a field of twisted cubics. This is known as a $\mathbf{G L}(2, \mathbb{R})$ structure on $X$. It was demonstrated by Bryant [8] that every four-dimensional $\mathbf{G L}(2, \mathbb{R})$ structure defines on $X$ a canonical affine connection which preserves the $\mathbf{G L}(2, \mathbb{R})$ structure, and whose torsion lies in 8-dimensional irreducible representation of $\mathbf{G L}(2, \mathbb{R})$. We will call it the Bryant connection, see Sect. 5.4 for computational formulae. Various important properties of system (1) have natural interpretation in terms of this connection:

- System (1) is linearly degenerate and integrable if and only if the Bryant connection is symmetric and flat (Proposition 5 of Sect. 4.2).

- System (1) is integrable if and only if the curvature $R$ and the covariant derivative $\nabla T$ of the torsion $T$ of the Bryant connection are certain invariant quadratic expressions in $T$,

$$
R=f\left(T^{2}\right), \quad \nabla T=g\left(T^{2}\right),
$$

see Theorem 3 of Sect. 5.4 for precise statements. These expressions are analogous to the ones obtained by Smith [56] in the context of 5-dimensional $\mathbf{G L}(2, \mathbb{R})$ structures associated with integrable equations of the dispersioless Hirota type [27]. Our expressions provide a compact invariant formulation of the integrability conditions.

\subsection{The method of hydrodynamic reductions}

In the most general set-up, the method of hydrodynamic reductions [24] applies to quasilinear systems of the form

$$
A(\mathbf{u}) \mathbf{u}_{x}+B(\mathbf{u}) \mathbf{u}_{y}+C(\mathbf{u}) \mathbf{u}_{t}=0,
$$

where $\mathbf{u}=\left(u^{1}, \ldots, u^{m}\right)^{t}$ is an $m$-component column vector of the dependent variables, and $A, B, C$ are $l \times m$ matrices where $l$, the number of equations, is allowed to exceed the number of unknowns, $m$. Note that system (1) can be brought to form (5) by choosing $u_{i}, v_{i}$ as the new dependent variables and writing out all possible consistency conditions among them - see Sect. 2.1 for details. The method of hydrodynamic reductions consists of seeking multiphase solutions in the form

$$
\mathbf{u}=\mathbf{u}\left(R^{1}, \ldots, R^{N}\right)
$$


where the phases $R^{i}(x, y, t)$, whose number $N$ is allowed to be arbitrary, are required to satisfy a pair of consistent $(1+1)$-dimensional systems,

$$
R_{t}^{i}=\lambda^{i}(R) R_{x}^{i}, \quad R_{y}^{i}=\mu^{i}(R) R_{x}^{i},
$$

known as systems of hydrodynamic type. The corresponding characteristic speeds $\lambda^{i}$ and $\mu^{i}$ are required to satisfy the commutativity conditions [58],

$$
\frac{\partial_{j} \lambda^{i}}{\lambda^{j}-\lambda^{i}}=\frac{\partial_{j} \mu^{i}}{\mu^{j}-\mu^{i}}
$$

here $i \neq j, \partial_{j}=\partial_{R^{j}}$. Solutions of this type originate from gas dynamics, and are known as nonlinear interactions of planar simple waves. They can be interpreted as natural dispersionless analogues of finite-gap solutions of $2+1$ dimensional soliton equations. Equations (6) are said to define an $N$-component hydrodynamic reduction of the original system (5). System (5) is said to be integrable if, for every $N$, it possesses infinitely many $N$-component hydrodynamic reductions parametrised by $N$ arbitrary functions of one variable. This requirement imposes strong constraints (integrability conditions) on the matrix elements of $A, B$ and $C$.

One can show that the existence of 3 -component reductions is already sufficiently restrictive and implies the existence of $N$-component reductions for arbitrary $N$. Thus, one can define integrability as the existence of infinitely many 3-component reductions parametrised by 3 arbitrary functions of one variable. This property is reminiscent of the well-known 3-soliton condition in the theory of $(1+1)$-dimensional integrable soliton equations. We refer to [24] for further details and references.

\subsection{Summary of the main results}

In Sect. 2.1 we apply the method of hydrodynamic reductions to equations of type (1). This results in an overdetermined involutive system of PDEs for the functions $F$ and $G$ (the integrability conditions). The analysis of this system leads to our first result (Theorem 1 of section 2.1 and Proposition 2 of section 3):

The moduli space of non-degenerate integrable systems (1) is 6-dimensional. Furthermore, the following conditions are equivalent:

(a) System (1) is integrable by the method of hydrodynamic reductions.

(b) Conformal structure $g$ defined by the characteristic variety of system (1) is Einstein-Weyl on every solution (with covector $\omega$ given by formula (4)).

Thus, Einstein-Weyl equations (3) provide an efficient integrability test. Using the integrability conditions we prove that every integrable system (1) possesses a dispersionless Lax pair (Theorem 2 of Sect. 2.2). Furthermore, we describe a construction that links Lax pairs to null totally geodesic surfaces of the corresponding Einstein-Weyl structure (Sect. 2.3).

In Sect. 3 we demonstrate that the class of equations (1) is invariant under the equivalence group $\mathbf{S L}(5)$ that acts by linear transformations on the combined set of variables $x^{1}, x^{2}, x^{3}, u, v$. All our results are formulated modulo this equivalence.

In Sect. 4 we describe a variety of integrable examples of type (1) expressible in elementary functions, modular forms and theta functions. In particular, we demonstrate that system (1) is linearisable by a transformation from the equivalence group if and only if the conformal structure defined by its characteristic variety is flat on every solution (Sect. 4.1). Next, we characterise linearly degenerate integrable systems in terms of the associated Bryant connection. Fourfolds 
$X$ corresponding to such systems are images of quadratic maps $\mathbb{P}^{4} \rightarrow \mathbf{G r}(3,5)$ given by the classical construction of Chasles. This provides a complete list of normal forms of linearly degenerate integrable systems (Sect. 4.2). Based on the construction of Odesskii and Sokolov [45, 46] we point out that generic integrable systems (1) can be parametrised by generalised hypergeometric functions (Sect. 4.4). This demonstrates that fourfolds $X \subset \mathbf{G r}(3,5)$ corresponding to integrable systems (1) may have a fairly intricate analytic structure: in particular, they do not need to be algebraic. Among the simplest nontrivial examples of this kind one can mention the system

$$
u_{t}=v_{x}, \quad v_{t}=\frac{u_{y}}{v_{x}}+\frac{1}{6} \eta\left(u_{x}\right) v_{x}^{2}
$$

which appeared in [47] in the classification of integrable hydrodynamic chains. In this case the integrability conditions result in the Chazy equation for $\eta, \eta^{\prime \prime \prime}+2 \eta \eta^{\prime \prime}-3 \eta^{\prime 2}=0$ (here prime denotes differentiation by $u_{x}$ ), whose generic solution is expressible in terms of the Eisenstein series $E_{2}$.

In Sect. 5 we demonstrate that every fourfold $X \subset \mathbf{G r}(3,5)$ inherits a $\mathbf{G L}(2, \mathbb{R})$ structure, namely, a field of twisted cubics specified in the projectivised tangent bundle PTX (Sect. 5.1). The integrability can be reformulated geometrically as the requirement that the associated $\mathbf{G L}(2, \mathbb{R})$ structure possesses infinitely many three-dimensional submanifolds carrying a holonomic characteristic net (Sect. 5.2). We show that every fourfold $X$ corresponding to integrable system (1) carries canonical conformally symplectic structure which is parallel in the Bryant connection (Sect. 5.3). Finally, we reformulate the integrability conditions of system (1) in terms of the curvature and torsion of the associated connection (Theorem 3 of Sect. 5.4). We emphasize that $\mathbf{G L}(2, \mathbb{R})$ structures arising in this context are quite different from those discussed in [8], in particular, they generally have nonzero torsion.

Throughout the paper, most of our considerations are local, although some results related to algebraic fourfolds $X \subset \mathbf{G r}(3,5)$ have global nature. We are primarily interested in real smooth structures and hyperbolic systems, however, the theory applies to the complex-analytic case as well (in particular, our classification results are obtained over $\mathbb{C}$ ). In the calculations we use computer algebra systems Mathematica and Maple (these only utilise symbolic polynomial algebra over $\mathbb{Q}$, so the results are rigorous). The programs are available from arXiv:1503.02274v1 [math.DG].

\section{Integrability conditions and Lax pairs}

\subsection{Derivation of the integrability conditions}

In this section we establish our first main result:

Theorem 1 The parameter space of non-degenerate integrable systems (1) is 30-dimensional. Furthermore, the following conditions are equivalent:

(a) System (1) is integrable by the method of hydrodynamic reductions.

(b) Conformal structure $g$ defined by the characteristic variety of system (1) is Einstein-Weyl on every solution (with covector $\omega$ given by formula (4)).

\section{Proof:}

Applied to system (1), the method of hydrodynamic reductions leads to a set of differential constraints (integrability conditions) for the functions $F$ and $G$. The 30-dimensionality of 
the parameter space follows from the involutivity of these conditions. Once the integrability conditions are derived, the equivalence of (a) and (b) can be shown by a direct calculation. Alternatively, one can utilise the fact that every integrable system (1) possesses a dispersionless Lax pair (Theorem 2 of Sect. 2.2), which provides null totally geodesic surfaces of the corresponding Einstein-Weyl structure $(\mathbb{D}, g, \omega)$. This implies the Einstein-Weyl property due to the result of Cartan [12].

To derive the integrability conditions we proceed as follows. Let us first rewrite system (1) in evolutionary form,

$$
u_{t}=f\left(u_{x}, u_{y}, v_{x}, v_{y}\right), \quad v_{t}=g\left(u_{x}, u_{y}, v_{x}, v_{y}\right),
$$

here we use the notation $\left(x^{1}, x^{2}, x^{3}\right)=(x, y, t)$. Our strategy is to derive a set of constraints for the right hand sides $f$ and $g$ that are necessary and sufficient for integrability. We begin by transforming our system into first-order quasilinear form. This can be done by taking firstorder partial derivatives of $u$ and $v$ as the new dependent variables and writing out all possible consistency conditions among them. Thus, we introduce the notation

$$
u_{x}=a, u_{y}=b, v_{x}=p, v_{y}=q, u_{t}=f(a, b, p, q), v_{t}=g(a, b, p, q) .
$$

This provides an equivalent quasilinear representation,

$$
\begin{aligned}
& a_{y}=b_{x}, \quad a_{t}=f(a, b, p, q)_{x}, \quad b_{t}=f(a, b, p, q)_{y}, \\
& p_{y}=q_{x}, \quad p_{t}=g(a, b, p, q)_{x}, \quad q_{t}=g(a, b, p, q)_{y} .
\end{aligned}
$$

Note that quasilinear representation (9) is of type (5) with $m=4, l=6, \mathbf{u}=(a, b, p, q)$. Looking for multi-phase solutions in the form

$$
a=a\left(R^{1}, \ldots, R^{N}\right), b=b\left(R^{1}, \ldots, R^{N}\right), p=p\left(R^{1}, \ldots, R^{N}\right), q=q\left(R^{1}, \ldots, R^{N}\right),
$$

where the phases $R^{i}$ satisfy equations (6), and substituting this ansatz into (9), we obtain the relations

$$
\partial_{i} b=\mu^{i} \partial_{i} a, \quad \partial_{i} q=\mu^{i} \partial_{i} p,
$$

here $\partial_{i}=\partial_{R^{i}}$, as well as

$$
\left(\lambda^{i}-f_{a}-\mu^{i} f_{b}\right) \partial_{i} a=\left(f_{p}+\mu^{i} f_{q}\right) \partial_{i} p, \quad\left(\lambda^{i}-g_{p}-\mu^{i} g_{q}\right) \partial_{i} p=\left(g_{a}+\mu^{i} g_{b}\right) \partial_{i} a .
$$

The last two equations imply the dispersion relation connecting $\lambda^{i}$ and $\mu^{i}$,

$$
\left(\lambda^{i}-f_{a}-\mu^{i} f_{b}\right)\left(\lambda^{i}-g_{p}-\mu^{i} g_{q}\right)=\left(f_{p}+\mu^{i} f_{q}\right)\left(g_{a}+\mu^{i} g_{b}\right) .
$$

In what follows we assume that the dispersion relation defines an irreducible conic in the $(\lambda, \mu)$ plane: this is equivalent to the non-degeneracy of system (1) as discussed in Sect. 1.2. Setting in (12) $\partial_{i} a=\varphi^{i} \partial_{i} p$ and solving the resulting equations for $\lambda^{i}$ and $\mu^{i}$ we can parametrise dispersion relation (13) in the form

$$
\mu^{i}=-\frac{f_{p}+\left(f_{a}-g_{p}\right) \varphi^{i}-g_{a} \varphi^{i^{2}}}{f_{q}+\left(f_{b}-g_{q}\right) \varphi^{i}-g_{b} \varphi^{i^{2}}}, \quad \lambda^{i}=\frac{\left(f_{q}+f_{b} \varphi^{i}\right)\left(g_{p}+g_{a} \varphi^{i}\right)-\left(f_{p}+f_{a} \varphi^{i}\right)\left(g_{q}+g_{b} \varphi^{i}\right)}{f_{q}+\left(f_{b}-g_{q}\right) \varphi^{i}-g_{b} \varphi^{i^{2}}} .
$$


Substituting these parametric expressions into the commutativity conditions (7), and using the relations

$$
\partial_{i} a=\varphi^{i} \partial_{i} p, \quad \partial_{i} b=\mu^{i} \varphi^{i} \partial_{i} p, \quad \partial_{i} q=\mu^{i} \partial_{i} p,
$$

we obtain $\partial_{j} \varphi^{i}$ in the form $\partial_{j} \varphi^{i}=(\ldots) \partial_{j} p, i \neq j$, where dots denote rational expressions in $\varphi^{i}, \varphi^{j}$ whose coefficients depend on second-order partial derivatives of $f$ and $g$ (we omit these expressions due to their complexity). Calculating consistency conditions for relations (14), $\partial_{i} \partial_{j} a=\partial_{j} \partial_{i} a, \partial_{i} \partial_{j} b=\partial_{j} \partial_{i} b, \partial_{i} \partial_{j} q=\partial_{j} \partial_{i} q$, we obtain (one and the same!) expression for $\partial_{i} \partial_{j} p$ in the form $\partial_{i} \partial_{j} p=(\ldots) \partial_{i} p \partial_{j} p, i \neq j$, where, again, dots denote terms rational in $\varphi^{i}$ and $\varphi^{j}$. Ultimately, $N$-phase solutions are governed by the relations

$$
\partial_{j} \varphi^{i}=(\ldots) \partial_{j} p, \quad \partial_{i} \partial_{j} p=(\ldots) \partial_{i} p \partial_{j} p, \quad i \neq j .
$$

To ensure their solvability we need to impose the compatibility conditions

$$
\partial_{k} \partial_{j} \varphi^{i}=\partial_{j} \partial_{k} \varphi^{i}, \quad \partial_{k} \partial_{j} \partial_{i} p=\partial_{j} \partial_{k} \partial_{i} p,
$$

that are required to hold for every triple of indices $i \neq j \neq k$. Direct calculation based on (14) and (15) results in

$$
\partial_{k} \partial_{j} \varphi^{i}-\partial_{j} \partial_{k} \varphi^{i}=(\ldots) \partial_{j} p \partial_{k} p, \quad \partial_{k} \partial_{j} \partial_{i} p-\partial_{j} \partial_{k} \partial_{i} p=(\ldots) \partial_{i} p \partial_{j} p \partial_{k} p,
$$

where dots denote rational expressions in $\varphi^{i}, \varphi^{j}, \varphi^{k}$ whose coefficients depend on partial derivatives of $f$ and $g$ up to order three. To ensure solvability of equations (15) we set all these coefficients equal to zero. This gives a system of differential constraints for $f$ and $g$ that is linear in the third-order derivatives thereof. Moreover, the number of linearly independent compatibility conditions equals the total number of third-order partial derivatives of $f$ and $g$, namely 40. Solving for third-order derivatives of $f$ and $g$ we obtain the required integrabililty conditions that can be represented in symbolic form,

$$
d^{3} f=R\left(d f, d g, d^{2} f, d^{2} g\right), \quad d^{3} g=S\left(d f, d g, d^{2} f, d^{2} g\right),
$$

40 equations altogether. Here $R$ and $S$ depend rationally on the first- and second-order partial derivatives of $f$ and $g$. Straightforward calculation shows that overdetermined system (17) is in involution. Thus, the parameter space of integrable systems (1) is 30-dimensional: one can arbitrarily specify the values of $f, g, d f, d g$ and $d^{2} f, d^{2} g$ at any fixed point. This amounts to $2 \times 1+2 \times 4+2 \times 10=30$ arbitrary constants.

Finally, the equivalence of (a) and (b) can be established by a direct calculation: in coordinates $(x, y, t)$, the characteristic variety of system (8) defines contravariant metric (metric with upper indices) with the matrix

$$
g^{i j}=\left(\begin{array}{ccc}
f_{a} g_{p}-f_{p} g_{a} & \frac{1}{2}\left(f_{a} g_{q}-f_{q} g_{a}+f_{b} g_{p}-f_{p} g_{b}\right) & -\frac{1}{2}\left(f_{a}+g_{p}\right) \\
\frac{1}{2}\left(f_{a} g_{q}-f_{q} g_{a}+f_{b} g_{p}-f_{p} g_{b}\right) & f_{b} g_{q}-f_{q} g_{b} & -\frac{1}{2}\left(f_{b}+g_{q}\right) \\
-\frac{1}{2}\left(f_{a}+g_{p}\right) & -\frac{1}{2}\left(f_{b}+g_{q}\right) & 1
\end{array}\right) .
$$

Introducing $\omega$ by formula (4) and calculating Einstein-Weyl conditions (3) we obtain expressions depending on third-order partial derivatives of $u$ and $v$. These have to vanish identically modulo (8), in other words, on every solution. Using (8) and its differential consequences to eliminate 
all partial derivatives involving differentiation by $t$, we obtain differential expressions that are polynomial in the remaining second- and third-order partial derivatives of $u$ and $v$. It can be verified directly that the vanishing of all coefficients of these polynomials is equivalent to integrability conditions (17). More precisely, the Einstein-Weyl conditions contain terms of two types: linear in third-order derivatives, and quadratic in second-order derivatives of $u$ and $v$. Coefficients at third-order derivatives of $u$ and $v$ vanish identically due to the choice of $\omega$, while coefficients at quadratic terms (105 coefficients altogether) give all of the 40 integrability conditions (17). This finishes the proof of Theorem 1.

Remark. Although the computations described above are rather involved (and certainly not doable with pen and paper), the programs that perform them are straightforward. For instance, the program Int_3D-conditions that derives the integrability conditions can be readily checked: its steps follow the proof of Theorem 1.

\subsection{Dispersionless Lax pairs}

We say that system (1) possesses a dispersionless Lax pair if there exists an auxiliary function $S$ satisfying two Hamilton-Jacobi type equations,

$$
S_{2}=P\left(S_{1}, u_{i}, v_{i}\right), \quad S_{3}=Q\left(S_{1}, u_{i}, v_{i}\right),
$$

such that the compatibility condition, $S_{23}=S_{32}$, holds identically modulo (1); here $S_{i}=\partial S / \partial x^{i}$. Note that the dependence of $P$ and $Q$ on $S_{1}$ is usually nonlinear. Lax pairs of this type appeared in the context of dispersionless integrability in [62]; in many cases, including the one studied in this paper, the existence of a dispersionless Lax pair is equivalent to the integrability by the method of hydrodynamic reductions. For instance, system (2) possesses the Lax pair

$$
S_{y}=S_{x}^{2}+v_{x} S_{x}, \quad S_{t}=\frac{4}{3} S_{x}^{3}+2 v_{x} S_{x}^{2}+\left(u_{x}+v_{x}^{2}\right) S_{x}
$$

the compatibility condition $S_{y t}=S_{t y}$ is satisfied identically modulo (2). The main result of this section is the following:

Theorem 2 Integrability of system (1) is equivalent to the existence of a 'generic' dispersionless Lax pair.

The definition of the term 'generic' is given after formula (20).

\section{Proof:}

Let us again consider system (1) in evolutionary form,

$$
u_{t}=f\left(u_{x}, u_{y}, v_{x}, v_{y}\right), \quad v_{t}=g\left(u_{x}, u_{y}, v_{x}, v_{y}\right) .
$$

Setting $u_{x}=a, u_{y}=b, v_{x}=p, v_{y}=q$ we will look for a Lax pair in the form

$$
S_{y}=P\left(S_{x}, a, b, p, q\right), \quad S_{t}=Q\left(S_{x}, a, b, p, q\right) .
$$


Requiring that the consistency condition $S_{y t}=S_{t y}$ holds identically modulo (9), we obtain 6 first-order relations for $P$ and $Q$ :

$$
\begin{gathered}
f_{a} P_{a}+g_{a} P_{p}+P_{\lambda} Q_{a}-Q_{\lambda} P_{a}=0, \quad f_{p} P_{a}+g_{p} P_{p}+P_{\lambda} Q_{p}-Q_{\lambda} P_{p}=0, \\
Q_{a}=f_{b} P_{a}+f_{a} P_{b}+g_{b} P_{p}+g_{a} P_{q}+P_{\lambda} Q_{b}-Q_{\lambda} P_{b}, \\
Q_{p}=f_{q} P_{a}+f_{p} P_{b}+g_{q} P_{p}+g_{p} P_{q}+P_{\lambda} Q_{q}-Q_{\lambda} P_{q}, \\
Q_{b}=f_{b} P_{b}+g_{b} P_{q}, \quad Q_{q}=f_{q} P_{b}+g_{q} P_{q} ;
\end{gathered}
$$

here $\lambda=S_{x}$. We say that system (1) possesses a 'generic' Lax pair if relations (20) are in involution, and $P, Q$ do not satisfy any extra first-order relations other than (20). Generic Lax pair is a generic solution to (20). Differentiating each of these relations by $a, b, p, q$ and $\lambda$, we obtain 30 relations which, in the non-degenerate case, can be uniquely resolved for all secondorder partial derivatives of $P$ and $Q$, thus leading to a closed system. It can be verified directly that the resulting system is involutive if and only if the functions $f$ and $g$ satisfy integrability conditions (17). This finishes the proof of Theorem 2.

Remark 1. The general solution of the involutive system for $P$ and $Q$ depends on 6 arbitrary constants. This comes from the count $2+10-6=6$, where 2 corresponds to the values of $P$ and $Q, 10$ comes from the values of their first-order derivatives, and -6 is due to the 6 first-order relations (20). On the other hand, the Lax pair is form-invariant under transformations

$$
S \rightarrow \alpha S+\beta u+\gamma v+\mu x+\nu y+\eta t,
$$

which also involve 6 arbitrary constants. Modulo these transformations, 'generic' Lax pair is essentially unique.

Remark 2. One can show that, as a consequence of relations (20), the quantities $P_{\lambda}$ and $Q_{\lambda}$ satisfy the dispersion relation,

$$
\operatorname{det}\left[\left(\begin{array}{cc}
f_{a} & f_{p} \\
g_{a} & g_{p}
\end{array}\right)+\left(\begin{array}{cc}
f_{b} & f_{q} \\
g_{b} & g_{q}
\end{array}\right) P_{\lambda}-\left(\begin{array}{cc}
1 & 0 \\
0 & 1
\end{array}\right) Q_{\lambda}\right]=0
$$

This relation plays an important role in the geometric interpretation of Lax pairs discussed below.

\subsection{Lax pairs and null totally geodesic surfaces}

Here we outline a general construction that leads from Lax pair (19) to null totally geodesic surfaces of the corresponding Einstein-Weyl structure (DD, $g, \omega)$. Differentiating (19) by $x$ and setting $S_{x}=\lambda$ we obtain

$$
\lambda_{y}=P_{\lambda} \lambda_{x}+P_{a} a_{x}+P_{b} b_{x}+P_{p} p_{x}+P_{q} q_{x}, \quad \lambda_{t}=Q_{\lambda} \lambda_{x}+Q_{a} a_{x}+Q_{b} b_{x}+Q_{p} p_{x}+Q_{q} q_{x} .
$$

With this system we associate two vector fields,

$$
\begin{aligned}
& X=\frac{\partial}{\partial y}-P_{\lambda} \frac{\partial}{\partial x}+\left(P_{a} a_{x}+P_{b} b_{x}+P_{p} p_{x}+P_{q} q_{x}\right) \frac{\partial}{\partial \lambda} \\
& Y=\frac{\partial}{\partial t}-Q_{\lambda} \frac{\partial}{\partial x}+\left(Q_{a} a_{x}+Q_{b} b_{x}+Q_{p} p_{x}+Q_{q} q_{x}\right) \frac{\partial}{\partial \lambda}
\end{aligned}
$$


which live in the extended four-dimensional space with coordinates $x, y, t, \lambda$. Note that the compatibility condition, $\lambda_{y t}=\lambda_{t y}$, is equivalent to the commutativity of these vector fields: $[X, Y]=0$. The geometry behind this construction is as follows. Let us consider the cotangent bundle $Z$ of a solution $u(x, y, t), v(x, y, t)$, with local coordinates $\left(x, y, t, S_{x}, S_{y}, S_{t}\right)$. Equations (19) specify a four-dimensional submanifold $M^{4} \subset Z$ parametrised by $x, y, t$ and $\lambda$. The compatibility of equations (19) indicates that this submanifold is coisotropic. Vector fields $X, Y$ generate the kernel of the restriction to $M^{4}$ of the symplectic form $d S_{x} \wedge d x+d S_{y} \wedge d y+d S_{t} \wedge d t$. Equations (22) mean that $X, Y$ are tangential to the Lagrangian submanifold of $M^{4}$ defined by the equation $\lambda=\lambda(x, y, t)$. Solutions $S(x, y, t)$ of equations (19) can be interpreted as generating functions of Lagrangian submanifolds contained in $M^{4}$. Such submanifolds depend on one arbitrary function of a single variable; they can be obtained by taking any one-parameter subfamily of the two-parameter family of integral surfaces of the distribution $\langle X, Y\rangle$ foliating $M^{4}$.

Projecting the two-parameter family of integral surfaces of the distribution $\langle X, Y\rangle$ from $M^{4}$ to the space of independent variables $x, y, t$ we obtain a two-parameter family of null totally geodesic surfaces of the Weyl connection $\mathbb{D}$. Indeed, projecting $X$ and $Y$ gives vector fields

$$
\hat{X}=\frac{\partial}{\partial y}-P_{\lambda} \frac{\partial}{\partial x}, \quad \hat{Y}=\frac{\partial}{\partial t}-Q_{\lambda} \frac{\partial}{\partial x},
$$

which commute if and only if $\lambda$ satisfies equations (22). It remains to show that $\langle\hat{X}, \hat{Y}\rangle$ is a null distribution (that is, tangential to the null cones of $g$ ), and that the covariant derivatives $\mathbb{D}_{\hat{X}} \hat{X}, \mathbb{D}_{\hat{X}} \hat{Y}, \mathbb{D}_{\hat{Y}} \hat{X}, \mathbb{D}_{\hat{Y}} \hat{Y}$ belong to $\langle\hat{X}, \hat{Y}\rangle$. Equivalently, one can introduce the covector $\theta=$ $d x+P_{\lambda} d y+Q_{\lambda} d t$ that annihilates $\hat{X}, \hat{Y}$, and verify that $\theta$ is null, and that $\mathbb{D}_{\hat{X}} \theta \wedge \theta=\mathbb{D}_{\hat{Y}} \theta \wedge \theta=0$. This follows from equations (20) satisfied by the functions $P(\lambda, a, b, c)$ and $Q(\lambda, a, b, c)$. In particular, the fact that $\theta$ is null follows from identity (21).

\section{$3 \mathrm{SL}(5)$ as the equivalence group: geometry of $\operatorname{Gr}(3,5)$}

Let $V^{5}$ be 5 -dimensional vector space with coordinates $x^{1}, x^{2}, x^{3}, u, v$, set $\mathbf{p}=(u, v)^{t}$ and $\mathbf{x}=$ $\left(x^{1}, x^{2}, x^{3}\right)^{t}$. Three-dimensional linear subspaces of $V$ are defined by the equation $d \mathbf{p}=U d \mathbf{x}$ where

$$
U=\left(\begin{array}{lll}
u_{1} & u_{2} & u_{3} \\
v_{1} & v_{2} & v_{3}
\end{array}\right)
$$

Thus, the Grassmannian $\mathbf{G r}(3,5)$ is six-dimensional, with an affine chart identified with the space of $2 \times 3$ matrices $U$. Solutions to system (1), $u\left(x^{1}, x^{2}, x^{3}\right)$ and $v\left(x^{1}, x^{2}, x^{3}\right)$, can be interpreted as three-dimensional submanifolds of $V^{5}$. Their tangent spaces are specified by matrices $U$ whose entries coincide with first-order derivatives of $u$ and $v: u_{i}=\partial u / \partial x^{i}, v_{i}=$ $\partial v / \partial x^{i}$. Thus, system (1) defines a fourfold $X \subset \mathbf{G r}(3,5)$, while solutions to (1) correspond to three-dimensional submanifolds of $V^{5}$ whose Gaussian images are contained in $X$. The action of $\mathbf{S L}(5)$,

$$
\left(\begin{array}{c}
d \tilde{\mathbf{p}} \\
d \tilde{\mathbf{x}}
\end{array}\right)=\left(\begin{array}{cc}
A & B \\
C & D
\end{array}\right)\left(\begin{array}{c}
d \mathbf{p} \\
d \mathbf{x}
\end{array}\right)
$$

naturally extends to $\mathbf{G r}(3,5)$ :

$$
\tilde{U}=(A U+B)(C U+D)^{-1}
$$


here $A, B, C, D$ are $2 \times 2,2 \times 3,3 \times 2$ and $3 \times 3$ matrices, respectively; notice that the extended action is no longer linear. Transformation law (23) suggests that the action of SL(5) preserves the class of equations (1), indeed, first-order derivatives transform through first-order derivatives only. Transformations of this form preserve integrability, so that $\mathbf{S L}(5)$ can be viewed as the natural equivalence group of the problem: it maps integrable systems to integrable systems. Thus, SL(5) is the point symmetry group of integrability conditions (17) derived in Sect. 2.1. The classification of integrable systems (1) will be performed modulo this equivalence: two SL(5)-related equations should be regarded as 'the same'.

Geometrically, our problem is reduced to the classification of fourfolds $X$ of the Grassmannian $\mathbf{G r}(3,5)$ that satisfy certain 'integrability' conditions (to be specified later, see Sect. 5.2), modulo the action of $\mathbf{S L}(5)$.

Given two infinitesimally close three-dimensional linear subspaces of $V^{5}$ defined by $2 \times 3$ matrices $U$ and $U+d U$, the condition that their intersection is two-dimensional is given by rank $d U=1$. This condition is invariant under the action of $\mathbf{S L}(5)$ as specified by (23). Thus, each tangent space of $\mathbf{G r}(3,5)$ is equipped with the cone $C$ defined by the equation $\operatorname{rank} d U=1$, that is, by the vanishing of all $2 \times 2$ minors of $d U$. Projectivisation of this cone is known as the Segre variety; it is a non-singular algebraic threefold of degree three. The field of Segre cones supplies $\mathbf{G r}(3,5)$ with the generalised flat conformal structure; it is manifestly invariant under the action of $\mathbf{S L}(5)$. The converse is also true: transformations from $\mathbf{S L}(5)$ are the only diffeomorphisms that preserve the field of Segre cones.

Proposition 1. The group of conformal automorphisms of the field of Segre cones is isomorphic to $\mathbf{S L}(5)$.

This is a well-known fact: direct proof would consist of the calculation of conformal automorphisms of the family of Segre cones. Let us point out that, in coordinates $u_{i}, v_{i}$, the infinitesimal generators corresponding to equivalence transformations (23) are as follows:

6 translations:

$$
\mathbf{U}_{i}=\frac{\partial}{\partial u_{i}}, \quad \mathbf{V}_{i}=\frac{\partial}{\partial v_{i}},
$$

12 linear transformations (note the relation $\sum \mathbf{X}_{i i}=\mathbf{L}_{11}+\mathbf{L}_{22}$ ):

$$
\mathbf{X}_{i j}=u_{i} \frac{\partial}{\partial u_{j}}+v_{i} \frac{\partial}{\partial v_{j}}, \quad \mathbf{L}_{11}=u_{k} \frac{\partial}{\partial u_{k}}, \quad \mathbf{L}_{12}=u_{k} \frac{\partial}{\partial v_{k}}, \quad \mathbf{L}_{21}=v_{k} \frac{\partial}{\partial u_{k}}, \quad \mathbf{L}_{22}=v_{k} \frac{\partial}{\partial v_{k}} .
$$

6 non-linear (projective) transformations:

$$
\mathbf{P}_{i}=u_{i} u_{k} \frac{\partial}{\partial u_{k}}+v_{i} u_{k} \frac{\partial}{\partial v_{k}}, \quad \mathbf{Q}_{i}=u_{i} v_{k} \frac{\partial}{\partial u_{k}}+v_{i} v_{k} \frac{\partial}{\partial v_{k}},
$$

The ideal $\Omega$ defining the field of Segre cones is generated by quadratic forms,

$$
\Omega=\operatorname{span}\left\{d u_{i} d v_{j}-d v_{i} d u_{j}\right\}
$$

which are nothing but second fundamental forms of the Plücker embedding of the Grassmannian, see e.g. [35]. It remains to point out that any vector field $X$ satisfying $L_{X} \Omega=0(\bmod \Omega)$ is spanned by the above infinitesimal generators. We refer to $[34,4,32,33]$ for generalisations of this Liouville-type result. 
Consider the action of the equivalence group $\mathbf{S L}(5)$ on the space $J^{1}\left(\mathbb{R}^{4}, \mathbb{R}^{2}\right)$ of 1-jets of functions $f, g$ of variables $a, b, p, q$. This is a 14-dimensional space with coordinates $u_{i}, v_{i}, f, g, f_{u_{i}}, f_{v_{i}}$, $g_{u_{i}}, g_{v_{i}}, i=1,2$, which can be viewed as an affine chart in the bundle of 4-dimensional tangent subspaces of $\mathbf{G r}(3,5)$. The action of $\mathbf{S L}(5)$ is canonically defined in the latter space, but by abuse of notation we will be working with $J^{1}$.

Lemma. The group $\mathbf{S L}(5)$ has a unique Zariski open orbit in $J^{1}\left(\mathbb{R}^{4}, \mathbb{R}^{2}\right)$ (its complement consists of 1-jets of degenerate systems).

The proof of this statement is as follows. The group $\mathbf{S L}(5)$ acts transitively on the Grassmannian $\mathbf{G r}(3,5)$ with the stabilizer of a point $o$ being the parabolic subgroup $P_{o}=S(\mathbf{G L}(2) \times$ $\mathbf{G L}(3)) \ltimes\left(\mathbb{R}^{2} \otimes \mathbb{R}^{3}\right)$ of upper-triangular block matrices of the size $2+3$. As only $S(\mathbf{G L}(2) \times \mathbf{G L}(3))$ acts on $T_{o} \mathbf{G r}(3,5)$, (i.e. $\mathbb{R}^{2} \otimes \mathbb{R}^{3}$ acts trivially), the action is transitive on 4 -planes corresponding to non-degenerate 1-jets of $f, g$. At the level of Lie algebra $\mathfrak{s l}(5)$, prolongation of the above infinitesimal generators to $J^{1}\left(\mathbb{R}^{4}, \mathbb{R}^{2}\right)$ has full rank in the Zariski open set of non-degenerate 1-jets. Indeed, the $24 \times 14$ matrix of coefficients of these vector fields drops rank precisely on the submanifold $\operatorname{det} g^{i j}=0$ where $g^{i j}$ is the conformal structure defined by the characteristic variety.

Remark 1. The above lemma allows one to assume that all sporadic factors depending on first-order derivatives of $f$ and $g$ that arise in the process of Gaussian elimination in the proofs of our main results, are nonzero. This considerably simplifies the arguments by eliminating unessential branching. Furthermore, in the verification of polynomial identities involving first and second-order partial derivatives of $f$ and $g$ one can, without any loss of generality, give the first-order derivatives any 'generic' numerical values: this often renders otherwise impossible computations manageable.

In fact, even the action of the subgroup $\mathbf{S L}(3) \subset P_{o}$ is already transitive on the Grassmannian of nondegenerate 4-planes in $T_{o} \mathbf{G r}(3,5)$, and the stabilizer of such a 4-plane is the subgroup $H_{o}=\mathbf{G L}(2) \ltimes\left(\mathbb{R}^{2} \otimes \mathbb{R}^{3}\right)$ of $P_{o}$, where we identify $\mathbb{R}^{3} \simeq S^{2}\left(\mathbb{R}^{2}\right)$ as an $\mathbf{S L}(2)$-representation. Prolongation of this action to $J^{2}\left(\mathbb{R}^{4}, \mathbb{R}^{2}\right)$ is locally free near a generic point (straightforward calculation shows that the prolongation of infinitesimal generators of $\mathfrak{s l}(5)$ to the space of 2 -jets has full rank 24 at generic points).

Proposition 2. The action of the equivalence group $\mathbf{S L}(5)$ on the 30-dimensional parameter space of integrable systems (1) is locally free (on the Zariski open set of generic points).

That is, 'generic' orbits of this action are 24-dimensional, so that 'generic' integrable systems of type (1) do not possess any continuous point symmetries coming from the equivalence group. Thus, the moduli space of integrable systems depends on $30-24=6$ essential parameters. To prove the above statement let us note that the submanifold in the space of 3-jets of $f, g$ given by integrability conditions (17) projects diffeomorphically onto the space of 2-jets $J^{2}\left(\mathbb{R}^{4}, \mathbb{R}^{2}\right)$ (which has dimension 30).

Remark 2. The action of $\mathbf{S L}(5)$ on the 30 -dimensional parameter space $M^{30}$ is algebraic, so that by the Rosenlicht theorem [52] there exists a quotient $M_{\text {reg }}^{30} / \mathbf{S L}(5)$, which is a rational algebraic variety of dimension 6 (the geometric quotient $M^{30} / \mathbf{S L}(5)$ is singular). Indeed, the equivalence group transforms the initial conditions of system (19) rationally. 


\section{Examples and classification results}

This section contains various classification results based on integrability conditions (17).

We produce an abundance of non-trivial examples of integrable systems of type (1), both known and new, expressible in elementary functions, theta functions, modular forms and generalised hypergeometric functions.

\subsection{Linearisable systems}

In this section we characterise systems (1) that can be linearised by a transformation from the equivalence group $\mathbf{S L}(5)$. Taking a linear system, say $u_{3}=v_{1}, v_{3}=u_{2}$, and applying transformations from the equivalence group, one obtains systems of Monge-Ampère type,

$$
\begin{aligned}
& a^{i j}\left(u_{i} v_{j}-u_{j} v_{i}\right)+b^{i} u_{i}+c^{i} v_{i}+m=0, \\
& \alpha^{i j}\left(u_{i} v_{j}-u_{j} v_{i}\right)+\beta^{i} u_{i}+\gamma^{i} v_{i}+\mu=0,
\end{aligned}
$$

where each equation represents a linear combination of minors of the $2 \times 3$ matrix

$$
U=\left(\begin{array}{lll}
u_{1} & u_{2} & u_{3} \\
v_{1} & v_{2} & v_{3}
\end{array}\right)
$$

Conversely, in three dimensions every system of this form is linearisable.

Proposition 3. For a non-degenerate system of type (1), the following conditions are equivalent:

(a) System is linearisable by a transformation from the equivalence group $\mathbf{S L}(5)$.

(b) System belongs to Monge-Ampère class (24).

(c) System is invariant under an 8-dimensional subgroup of $\mathbf{S L}(5)$.

(d) The characteristic variety defines conformal structure that is flat on every solution.

\section{Proof:}

equivalence $(a) \Longleftrightarrow(b)$ : One needs to show that taking a non-degenerate linear system, say $u_{3}=v_{1}, v_{3}=u_{2}$ (all non-degenerate linear systems of type (1) are $\mathbf{S L}(5)$-equivalent), and applying transformations from the equivalence group, one obtains all systems of Monge-Ampère type. The easiest way to see this is the following. First of all, any non-degenerate linear system is invariant under an 8-dimensional subgroup of $\mathbf{S L}(5)$; note that 8 is the maximal possible value for the dimension of the stabiliser of the action of the equivalence group on the space of fourfolds $X \subset \mathbf{G r}(3,5)$. The corresponding infinitesimal generators can be calculated using the standard machinery of group analysis. Thus, the linear system $u_{3}=v_{1}, v_{3}=u_{2}$ is invariant under the subgroup with 8 infinitesimal generators,

$$
\begin{aligned}
& \mathbf{U}_{1}, \quad \mathbf{V}_{2}, \quad \mathbf{U}_{2}+\mathbf{V}_{3}, \quad \mathbf{U}_{3}+\mathbf{V}_{1}, \\
& \mathbf{X}_{13}+2 \mathbf{X}_{32}+\mathbf{L}_{12}, \quad \mathbf{X}_{23}+2 \mathbf{X}_{31}+\mathbf{L}_{21}, \quad \mathbf{X}_{11}+\mathbf{X}_{22}+\mathbf{X}_{33}, \quad \mathbf{X}_{11}-\mathbf{X}_{22}+\mathbf{L}_{11},
\end{aligned}
$$

(we use the notation of Sect. 3). The Lie algebra is isomorphic to a semi-direct product $V_{3} \rtimes \mathfrak{g l}(2)$ where $V_{3} \simeq S^{3}\left(\mathbb{R}^{2}\right)$ is the irreducible 4-dimensional representation of $\mathfrak{s l}(2)$ (and hence of $\mathfrak{g l}(2)=\mathfrak{s l}(2) \oplus \mathbb{R}$, with non-trivial action of $\mathbb{R})$. Thus, applying to a linear system transformations from the equivalence group, one obtains a variety of systems depending on $24-8=16$ essential parameters. It remains to point out that the class of Monge-Ampère systems also depends on 16 essential parameters. 
equivalence $(a) \Longleftrightarrow(c)$ : The first implication follows from the fact that any non-degenerate linear system is invariant under an 8-dimensional subalgebra of SL(5). To establish the converse, let $G$ be the symmetry group of system (1). We can always assume that the point $o$, specified by $u_{i}=v_{i}=0$, belongs to the fourfold $X \subset \mathbf{G r}(3,5)$ corresponding to our system. Let $G_{o}$ be the stabiliser of this point in $G$. Note that $\operatorname{dim} G-\operatorname{dim} G_{o} \leq 4$, as $G$ takes $X$ to itself. The stabiliser $P$ of the point $o$ is spanned by infinitesimal generators $\mathbf{X}_{i j}, \mathbf{L}_{i j}, \mathbf{P}_{i}, \mathbf{Q}_{i}$. Since the system is non-degenerate, we can always bring it to a canonical form:

$$
u_{3}=v_{1}+o\left(u_{i}, v_{i}\right), \quad v_{3}=u_{2}+o\left(u_{i}, v_{i}\right) .
$$

This form (together with the point $o$ ) is stabilised by the following four elements of $P$ :

$$
\mathbf{X}_{13}+2 \mathbf{X}_{32}+\mathbf{L}_{12}, \quad \mathbf{X}_{23}+2 \mathbf{X}_{31}+\mathbf{L}_{21}, \quad \mathbf{X}_{11}+\mathbf{X}_{22}+\mathbf{X}_{33}, \quad \mathbf{X}_{11}-\mathbf{X}_{22}+\mathbf{L}_{11} .
$$

Thus, $\operatorname{dim} G_{o} \leq 4$ so that $\operatorname{dim} G \leq 8$. The equality holds only if $\operatorname{dim} G_{o}=4$. However, the generator $\mathbf{X}_{11}+\mathbf{X}_{22}+\mathbf{X}_{33}$ acts by non-trivial rescalings on terms of order 2 and higher in (25). Hence, for $\operatorname{dim} G_{o}=4$, all higher-order terms must vanish identically, leading to a linear system.

equivalence $(a) \Longleftrightarrow(d)$ : Representing system (1) in evolutionary form (8) and introducing the corresponding conformal structure $g$ (see the proof of Theorem 1 for explicit formulae), the condition responsible for conformal flatness in three dimensions is the vanishing of the Cotton tensor,

$$
\nabla_{r}\left(R_{p q}-\frac{1}{4} R g_{p q}\right)=\nabla_{q}\left(R_{p r}-\frac{1}{4} R g_{p r}\right),
$$

where $R_{p q}$ is the Ricci tensor, $R$ is the scalar curvature, and $\nabla$ denotes covariant differentiation in the Levi-Civita connection of $g$. Calculating (26) and using (8) and its differential consequences to eliminate all higher-order partial derivatives of $u$ and $v$ containing differentiation by $x^{3}$, we obtain expressions that have to vanish identically in the remaining higher-order derivatives (no more than fourth-order derivatives will occur in this calculation). In particular, equating to zero coefficients at fourth-order derivatives of $u$ and $v$, we obtain the system of second-order PDEs for $f$ and $g$ :

$$
\begin{gathered}
f_{u_{i} u_{i}}=\frac{2 g_{u_{i}}}{g_{v_{i}}-f_{u_{i}}} f_{u_{i} v_{i}}, \quad f_{v_{i} v_{i}}=\frac{2 f_{v_{i}}}{f_{u_{i}}-g_{v_{i}}} f_{u_{i} v_{i}} \\
f_{u_{i} u_{j}}=\frac{g_{u_{j}}}{g_{v_{i}}-f_{u_{i}}} f_{u_{i} v_{i}}+\frac{g_{u_{i}}}{g_{v_{j}}-f_{u_{j}}} f_{u_{j} v_{j}}, \quad f_{v_{i} v_{j}}=\frac{f_{v_{j}}}{f_{u_{i}}-g_{v_{i}}} f_{u_{i} v_{i}}+\frac{f_{v_{i}}}{f_{u_{j}}-g_{v_{j}}} f_{u_{j} v_{j}}, \\
f_{u_{i} v_{j}}+f_{u_{j} v_{i}}=\frac{f_{u_{j}}-g_{v_{j}}}{f_{u_{i}}-g_{v_{i}}} f_{u_{i} v_{i}}+\frac{f_{u_{i}}-g_{v_{i}}}{f_{u_{j}}-g_{v_{j}}} f_{u_{j} v_{j}}
\end{gathered}
$$

here $i, j$ take any values from 1 to 2 ; the equations for $g$ can be obtained by the simultaneous substitution $f \leftrightarrow g$ and $u \leftrightarrow v$. The general solution of system (27) leads to Monge-Ampère systems. Furthermore, modulo (27) all remaining components of the Cotton tensor vanish identically (in fact, this follows from the linearisability of Monge-Ampère systems in 3D). This finishes the proof of Proposition 3.

Remark. Equations of Monge-Ampère type have a clear geometric interpretation. Recall that the Grassmannian $\mathbf{G r}(3,5)$ is (locally) identified with the space of $2 \times 3$ matrices $U$. Minors of $U$ define the Plücker embedding of $\mathbf{G r}(3,5)$ into projective space $\mathbb{P}^{9}$. We identify $\mathbf{G r}(3,5)$ with the image of this embedding, which is a non-singular algebraic variety of degree five. Thus, Monge-Ampère systems correspond to sections of $\mathbf{G r}(3,5)$ by subspaces $\mathbb{P}^{7} \subset \mathbb{P}^{9}$. 
We emphasize that the linearisability of Monge-Ampère systems is an essentially threedimensional phenomenon: in higher dimensions there exist integrable non-linearisable examples of Monge-Ampère type, e.g.

$$
u_{2}-v_{1}=0, \quad u_{3} v_{4}-u_{4} v_{3}-1=0,
$$

which is equivalent to the Plebański first heavenly equation [50].

\subsection{Linearly degenerate systems}

From the point of view of their 'complexity', linearly degenerate systems come next after linearisable systems. In this section we obtain a complete list of normal forms of linearly degenerate integrable systems: all of them come from quadratic maps $\mathbb{P}^{4} \rightarrow \mathbf{G r}(3,5)$ given by the classical construction of Chasles.

The definition of linear degeneracy is inductive: a multi-dimensional system is said to be linearly degenerate (completely exceptional [6]) if all its traveling wave reductions to two dimensions are linearly degenerate. Thus, it is sufficient to define this concept in 2D case,

$$
u_{2}=f\left(u_{1}, v_{1}\right), \quad v_{2}=g\left(u_{1}, v_{1}\right) .
$$

Setting $u_{1}=a, v_{1}=p$ and differentiating by $x^{1}$ one can rewrite this system in two-component quasilinear form,

$$
a_{2}=f(a, p)_{1}, \quad p_{2}=g(a, p)_{1},
$$

or, in matrix notation,

$$
\left(\begin{array}{c}
a \\
p
\end{array}\right)_{2}=A\left(\begin{array}{c}
a \\
p
\end{array}\right)_{1}, \quad A=\left(\begin{array}{cc}
f_{a} & f_{p} \\
g_{a} & g_{p}
\end{array}\right)
$$

Recall that the matrix $A$ is said to be linearly degenerate if its eigenvalues (assumed real and distinct) are constant in the direction of the corresponding eigenvectors. Explicitly, $L_{r^{i}} \lambda^{i}=0$, no summation, where $L_{r^{i}}$ denotes Lie derivative in the direction of the eigenvector $r^{i}$, and $A r^{i}=\lambda^{i} r^{i}$. For quasilinear systems, the property of linear degeneracy is known to be related, under appropriate 'small norm' assumptions, to the impossibility of breakdown of smooth initial data, leading to global solvability of a generic Cauchy problem [53]. In terms of the original functions $f\left(u_{1}, v_{1}\right)$ and $g\left(u_{1}, v_{1}\right)$, the conditions of linear degeneracy reduce to a pair of secondorder differential constraints,

$$
\begin{aligned}
& \left(f_{u_{1}}-g_{v_{1}}\right) f_{u_{1} u_{1}}+2 g_{u_{1}} f_{u_{1} v_{1}}+g_{u_{1}} g_{v_{1} v_{1}}+f_{v_{1}} g_{u_{1} u_{1}}=0 \\
& \left(g_{v_{1}}-f_{u_{1}}\right) g_{v_{1} v_{1}}+2 f_{v_{1}} g_{u_{1} v_{1}}+f_{v_{1}} f_{u_{1} u_{1}}+g_{u_{1}} f_{v_{1} v_{1}}=0 .
\end{aligned}
$$

Equations (28) define a 2-parameter family of 2-dimensional linear subspaces of a vector space $V^{4}$ (on projectivisation, a congruence of lines in $\mathbb{P}^{3}$ ). Geometrically, equations (29) mean that focal surfaces of this congruence degenerate into curves [2]. We point out that system (29) is invariant under the natural action of the $2 \mathrm{D}$ equivalence group $\mathbf{S L}(4)$. Requiring that all traveling wave reductions of a multi-dimensional system to $2 \mathrm{D}$ are linearly degenerate in the above sense, we obtain differential characterisation of linear degeneracy: 
Proposition 4. Ad-dimensional system, $u_{d}=f\left(u_{i}, v_{i}\right), v_{d}=g\left(u_{i}, v_{i}\right), i=1, \ldots, d-1$, is linearly degenerate if and only if the functions $f$ and $g$ satisfy the relations

$$
\begin{aligned}
& \operatorname{Sym}_{\{i, j, k\}}\left(\left(f_{u_{k}}-g_{v_{k}}\right) f_{u_{i} u_{j}}+g_{u_{k}}\left(f_{u_{i} v_{j}}+f_{u_{j} v_{i}}\right)+f_{v_{k}} g_{u_{i} u_{j}}+g_{u_{k}} g_{v_{i} v_{j}}\right)=0, \\
& \operatorname{Sym}_{\{i, j, k\}}\left(\left(g_{v_{k}}-f_{u_{k}}\right) g_{v_{i} v_{j}}+f_{v_{k}}\left(g_{u_{i} v_{j}}+g_{u_{j} v_{i}}\right)+g_{u_{k}} f_{v_{i} v_{j}}+f_{v_{k}} f_{u_{i} u_{j}}\right)=0,
\end{aligned}
$$

where Sym denotes complete symmetrisation over $i, j, k \in\{1, \ldots, d-1\}$.

\section{Proof:}

Let us begin with the 3-dimensional case,

$$
u_{3}=f\left(u_{1}, u_{2}, v_{1}, v_{2}\right), \quad v_{3}=g\left(u_{1}, u_{2}, v_{1}, v_{2}\right) .
$$

Looking for a traveling wave reduction in the form

$$
u\left(x^{1}, x^{2}, x^{3}\right)=u\left(y^{1}, y^{2}\right)+y^{3}, \quad v\left(x^{1}, x^{2}, x^{3}\right)=v\left(y^{1}, y^{2}\right)+y^{4},
$$

where $y^{i}$ are arbitrary constant-coefficient linear forms in the independent variables $x^{k}$, one obtains a system of type (28) for $u\left(y^{1}, y^{2}\right)$ and $v\left(y^{1}, y^{2}\right)$. It is required to be linearly degenerate for every choice of linear forms $y^{i}$. This condition imposes a set of second-order differential constraints for $f$ and $g$,

$$
\begin{gathered}
\left(f_{u_{j}}-g_{v_{j}}\right) f_{u_{i} u_{i}}+2\left(f_{u_{i}}-g_{v_{i}}\right) f_{u_{i} u_{j}}+2 g_{u_{j}} f_{u_{i} v_{i}}+2 g_{u_{i}}\left(f_{u_{i} v_{j}}+f_{u_{j} v_{i}}\right)+ \\
f_{v_{j}} g_{u_{i} u_{i}}+2 f_{v_{i}} g_{u_{i} u_{j}}+g_{u_{j}} g_{v_{i} v_{i}}+2 g_{u_{i}} g_{v_{i} v_{j}}=0 \\
\left(g_{v_{j}}-f_{u_{j}}\right) g_{v_{i} v_{i}}+2\left(g_{v_{i}}-f_{u_{i}}\right) g_{v_{i} v_{j}}+2 f_{v_{j}} g_{u_{i} v_{i}}+2 f_{v_{i}}\left(g_{u_{i} v_{j}}+g_{u_{j} v_{i}}\right)+ \\
g_{u_{j}} f_{v_{i} v_{i}}+2 g_{u_{i}} f_{v_{i} v_{j}}+f_{v_{j}} f_{u_{i} u_{i}}+2 f_{v_{i}} f_{u_{i} u_{j}}=0
\end{gathered}
$$

(no summation), where $i, j \in\{1,2\}$ (8 relations altogether). Notice that the second half of these relations follows from the first one under the simultaneous substitution $f \leftrightarrow g, u \leftrightarrow v$. Relations (31) can be obtained from (30) by setting $k=j$. For $i=j=1$ relations (31) simplify to (29). Relations (31) can be derived in either of the two ways:

Method 1. Applying the full 3D equivalence group SL(5) to constraints (29) one gets 8 linearly independent relations that are equivalent to (31). One can show that the set of relations (31) is $\mathbf{S L}(5)$-invariant. This procedure generalises to higher dimensions in an obvious way. For instance, in 4D, applying transformations from SL(6) to constraints (29) one gets 20 linearly independent relations $(30), d=4$.

Method 2. Looking for particular traveling wave reductions of the form $u\left(x^{1}, x^{2}, x^{3}\right)=$ $u\left(y^{1}, y^{2}\right), v\left(x^{1}, x^{2}, x^{3}\right)=v\left(y^{1}, y^{2}\right)$ where $y^{1}=x^{1}+\lambda x^{2}, y^{2}=x^{3}$, one obtains the reduced system in the form

$$
u_{y^{2}}=F\left(u_{y^{1}}, v_{y^{1}}\right)=f\left(u_{y^{1}}, \lambda u_{y^{1}}, v_{y^{1}}, \lambda v_{y^{1}}\right), \quad v_{y^{2}}=G\left(u_{y^{1}}, v_{y^{1}}\right)=g\left(u_{y^{1}}, \lambda u_{y^{1}}, v_{y^{1}}, \lambda v_{y^{1}}\right) .
$$

Here $F$ and $G$ have to satisfy relations (29). Since $F_{u_{y^{1}}}=f_{u_{1}}+\lambda f_{u_{2}}, F_{u_{y^{1}} u_{y^{1}}}=f_{u_{1} u_{1}}+2 \lambda f_{u_{1} u_{2}}+$ $\lambda^{2} f_{u_{2} u_{2}}$, etc, both relations (29) will be polynomial of degree 3 in $\lambda$. Equating to zero coefficients 
of these polynomials we get all relations (31). This generalises to higher dimensions: thus, in $4 \mathrm{D}$, given a system

$$
u_{4}=f\left(u_{1}, u_{2}, u_{3}, v_{1}, v_{2}, v_{3}\right), \quad v_{4}=g\left(u_{1}, u_{2}, u_{3}, v_{1}, v_{2}, v_{3}\right),
$$

one looks for traveling wave reductions $u\left(x^{1}, x^{2}, x^{3}, x^{4}\right)=u\left(y^{1}, y^{2}\right), v\left(x^{1}, x^{2}, x^{3}, x^{4}\right)=v\left(y^{1}, y^{2}\right)$ where $y^{1}=x^{1}+\lambda x^{2}+\mu x^{3}, y^{2}=x^{4}$. For the reduced system, both relations (29) become polynomials of degree 3 in $\lambda$ and $\mu$, each having 10 coefficients. Equating them to zero we obtain 20 differential constraints (30) constituting conditions of linear degeneracy in 4D. This finishes the proof of Proposition 4.

Remark 1. Second-order relations (31) governing linearly degenerate systems in 3D are not in involution, and their prolongation implies all third-order integrability conditions (17). This requires differentiating equations (31) three times and solving for the fifth, fourth and third-order partial derivatives of $f$ and $g$ in terms of the first and second-order derivatives.

Proposition 5. The parameter space of linearly degenerate integrable systems of type (1) is 22-dimensional. Furthermore, the following conditions are equivalent:

(a) System (1) is linearly degenerate and integrable.

(b) There exists a unique flat symmetric connection on $X$ in which the associated $\mathbf{G L}(2, \mathbb{R})$ structure is parallel.

\section{Proof:}

One can verify that 8 constraints (31) are compatible with integrability conditions (17). Remembering that the parameter space of integrable systems (1) is 30-dimensional, we thus obtain a $30-8=22$-dimensional parameter space of linearly degenerate integrable systems.

The equivalence of (a) and (b) can be demonstrated as follows. Using parametric equations of the fourfold $X$ as in Theorem 1 ,

$$
u_{x}=a, u_{y}=b, v_{x}=p, v_{y}=q, u_{t}=f(a, b, p, q), v_{t}=g(a, b, p, q),
$$

we define cubic cones of the induced $\mathbf{G L}(2, \mathbb{R})$ structure as the intersection of three quadratic forms, $\omega^{\alpha}=0$, where

$$
\omega^{1}=d a d q-d b d p, \quad \omega^{2}=d a d g-d p d f, \quad \omega^{3}=d b d g-d q d f .
$$

Note that these quadratic forms are nothing but restrictions to $X$ of the second fundamental forms of the Grassmannian $\mathbf{G r}(3,5) \subset \mathbb{P}^{9}$. A connection $\nabla$ preserving this $\mathbf{G L}(2, \mathbb{R})$ structure is defined by the relations $\nabla \omega^{\alpha}=0 \bmod \left\langle\omega^{\beta}\right\rangle$. Under the assumption that $\nabla$ is symmetric these relations lead to a linear inhomogeneous system for the 40 Christoffel's symbols of $\nabla$. This system is uniquely solvable if and only if the functions $f$ and $g$ satisfy conditions of linear degeneracy (31). One can verify by direct calculation that the vanishing of the curvature of this symmetric connection is equivalent to integrability conditions (17). This finishes the proof of Proposition 5.

Our next goal is to provide a complete list of normal forms of linearly degenerate integrable systems. The key example generating an open part of the 22-dimensional parameter space is as follows:

Example. Let $a_{1}, a_{2}, a_{3}$ and $\tilde{a}_{1}, \tilde{a}_{2}, \tilde{a}_{3}$ be two triplets of numbers such that $a_{1}+a_{2}+a_{3}=0$ and $\tilde{a}_{1}+\tilde{a}_{2}+\tilde{a}_{3}=0$. One can show that the following system,

$$
a_{1} \tilde{a}_{2} u_{x} v_{y}-a_{2} \tilde{a}_{1} u_{y} v_{x}=0, \quad a_{1} \tilde{a}_{3} u_{x} v_{t}-a_{3} \tilde{a}_{1} u_{t} v_{x}=0,
$$


is linearly degenerate and integrable. Eliminating $v$ we obtain a second-order equation for $u$, $a_{1} u_{x} u_{y t}+a_{2} u_{y} u_{x t}+a_{3} u_{t} u_{x y}=0$. Similarly, eliminating $u$ we obtain the analogous equation for $v, \tilde{a}_{1} v_{x} v_{y t}+\tilde{a}_{2} v_{y} v_{x t}+\tilde{a}_{3} v_{t} v_{x y}=0$. This construction first appeared in [61] in the context of bi-Hamiltonian systems and Veronese webs in 3D, see also [22]. Rewriting system (32) in simplified form,

$$
u_{x} v_{y}=\alpha u_{y} v_{x}, \quad u_{x} v_{t}=\beta u_{t} v_{x},
$$

$\alpha, \beta=$ const, one can show that:

(i) Systems with generic values of $\alpha$ and $\beta$ are not $\mathbf{S L}(5)$-equivalent.

(ii) For generic choice of $\alpha$ and $\beta$ the corresponding system is invariant under a 4-dimensional subgroup of the equivalence group (with infinitesimal generators $\mathbf{X}_{11}, \mathbf{X}_{22}, \mathbf{X}_{33}, \mathbf{L}_{11}$ in the notation of Sect. 3, that form a Cartan subalgebra of $\mathbf{S L}(5)$ ), and hence generates a $24-4=20$ dimensional orbit.

Acting on $(\alpha, \beta)$-systems by transformations from the equivalence group one thus obtains a 22-parameter family generating an open part of the parameter space of linearly degenerate integrable systems.

All linearly degenerate integrable systems of type (1), including the above example, can be obtained from the following geometric construction. Consider projective space $\mathbb{P}^{4}$ with homogeneous coordinates $\xi=\left(\xi^{1}: \xi^{2}: \xi^{3}: \xi^{4}: \xi^{5}\right)$. Let $A$ be a projective automorphism of $\mathbb{P}^{4}$ defined by a $5 \times 5$ matrix from $\mathbf{S L}(5)$. Consider the family of lines $\overline{(\xi, \eta)}$ through $\xi$ and $\eta=\xi A$ (the locus of lines spanned by an argument and the value of a projective transformation was apparently first discussed by Chasles [13]; see also [14], p. 556). The Plücker coordinates $p^{i j}=\xi^{i} \eta^{j}-\xi^{j} \eta^{i}$ are the $2 \times 2$ minors of the matrix

$$
\left(\begin{array}{ccccc}
\xi^{1} & \xi^{2} & \xi^{3} & \xi^{4} & \xi^{5} \\
\eta^{1} & \eta^{2} & \eta^{3} & \eta^{4} & \eta^{5}
\end{array}\right)
$$

they define quadratic map from the family of lines $\overline{(\xi, \eta)}$ (which is itself isomorphic to $\mathbb{P}^{4}$ ) into the Grassmannian of lines in $\mathbb{P}^{4}$, that is, into $\mathbf{G r}(2,5)$. By duality, this gives a fourfold $X$ in $\mathbf{G r}(3,5)$, and the corresponding system (1). Fourfolds $X$ arising from this construction are images of quadratic maps $\mathbb{P}^{4} \rightarrow \mathbf{G r}(3,5) \subset \mathbb{P}^{9}$. Explicit parametric equations of $X$, as well as of the corresponding system (1), can be obtained from the factorised representation

$$
\left(\begin{array}{lllll}
\xi^{1} & \xi^{2} & \xi^{3} & \xi^{4} & \xi^{5} \\
\eta^{1} & \eta^{2} & \eta^{3} & \eta^{4} & \eta^{5}
\end{array}\right)=\left(\begin{array}{ll}
\xi^{1} & \xi^{2} \\
\eta^{1} & \eta^{2}
\end{array}\right)\left(\begin{array}{ccccc}
1 & 0 & u_{1} & u_{2} & u_{3} \\
0 & 1 & v_{1} & v_{2} & v_{3}
\end{array}\right)
$$

explicitly,

$$
u_{1}=\frac{p^{32}}{p^{12}}, u_{2}=\frac{p^{42}}{p^{12}}, u_{3}=\frac{p^{52}}{p^{12}}, v_{1}=\frac{p^{13}}{p^{12}}, v_{2}=\frac{p^{14}}{p^{12}}, v_{3}=\frac{p^{15}}{p^{12}} .
$$

In the (generic) diagonal case, $A=\operatorname{diag}\left(\lambda^{i}\right)$, this gives

$$
\begin{aligned}
& u_{1}=\frac{\lambda^{2}-\lambda^{3}}{\lambda^{2}-\lambda^{1}} \frac{\xi^{2} \xi^{3}}{\xi^{1} \xi^{2}}, \quad u_{2}=\frac{\lambda^{2}-\lambda^{4}}{\lambda^{2}-\lambda^{1}} \frac{\xi^{2} \xi^{4}}{\xi^{1} \xi^{2}}, \quad u_{3}=\frac{\lambda^{2}-\lambda^{5}}{\lambda^{2}-\lambda^{1}} \frac{\xi^{2} \xi^{5}}{\xi^{1} \xi^{2}}, \\
& v_{1}=\frac{\lambda^{3}-\lambda^{1}}{\lambda^{2}-\lambda^{1}} \frac{\xi^{1} \xi^{3}}{\xi^{1} \xi^{2}}, \quad v_{2}=\frac{\lambda^{4}-\lambda^{1}}{\lambda^{2}-\lambda^{1}} \frac{\xi^{1} \xi^{4}}{\xi^{1} \xi^{2}}, \quad v_{3}=\frac{\lambda^{5}-\lambda^{1}}{\lambda^{2}-\lambda^{1}} \frac{\xi^{1} \xi^{5}}{\xi^{1} \xi^{2}},
\end{aligned}
$$

leading to the $(\alpha, \beta)$-system, $u_{1} v_{2}=\alpha u_{2} v_{1}, u_{1} v_{3}=\beta u_{3} v_{1}$, with

$$
\alpha=\frac{\left(\lambda^{2}-\lambda^{4}\right)\left(\lambda^{3}-\lambda^{1}\right)}{\left(\lambda^{2}-\lambda^{3}\right)\left(\lambda^{4}-\lambda^{1}\right)}, \quad \beta=\frac{\left(\lambda^{2}-\lambda^{5}\right)\left(\lambda^{3}-\lambda^{1}\right)}{\left(\lambda^{2}-\lambda^{3}\right)\left(\lambda^{5}-\lambda^{1}\right)} .
$$


The corresponding fourfold $X$ coincides with the quadratic image of $\mathbb{P}^{4}$ defined by a linear system of quadrics that pass through 5 points in general position (in our parametrisation, quadrics $\xi^{i} \xi^{j}, i \neq j$, that pass through 5 base points each having only one nonzero coordinate $\xi$ ). We point out that smooth quadratic embeddings of projective spaces into Grassmannians were discussed in [55]. It is important to emphasize that our $X$ is not smooth: lines through any pair of base points in $\mathbb{P}^{4}$ correspond to singular points of $X$. In algebro-geometric language, quadratic maps from $\mathbb{P}^{n}$ to the Grassmannian of lines in $\mathbb{P}^{n}$ are given by rank 2 vector bundles $\mathrm{E}$ with the first Chern class equal to 2 , together with $\mathrm{n}+1$ sections that generate $\mathrm{E}$. In the Chasles construction, $E=\mathcal{O}(1)+\mathcal{O}(1)$, and the space of sections is the graph of the map $A: H^{0}(\mathcal{O}(1)) \rightarrow H^{0}(\mathcal{O}(1))$.

Table 1 below comprises a complete list of 7 canonical forms of linearly degenerate integrable systems (1) labelled by Segre types (essentially, Jordan normal forms) of the $5 \times 5$ matrices $A$ from the Chasles construction. Thus, type [3,2] denotes two Jordan blocks of size $3 \times 3$ and $2 \times 2$, type [5] - one $5 \times 5$ Jordan block, etc (to get canonical forms as presented in Table 1 we take $A$ in Jordan normal form, eliminate $\xi$ 's from (33), and use equivalence transformations to remove unessential parameters). The last column provides dimensions of stabilisers of the resulting systems under the action of the equivalence group $\mathbf{S L}(5)$.

Table 1: Canonical forms of linearly degenerate integrable systems

\begin{tabular}{|l|l|c|}
\hline Segre type & Canonical form & Dim(stab) \\
\hline$[1111]]$ & $\begin{array}{l}u_{1} v_{2}=\alpha u_{2} v_{1} \\
u_{1} v_{3}=\beta u_{3} v_{1}\end{array}$ & 4 \\
\hline$[2111]$ & $\begin{array}{l}u_{1} v_{2}-u_{2} v_{1}=v_{1} v_{2} \\
u_{1} v_{3}-u_{3} v_{1}=\alpha v_{1} v_{3}\end{array}$ & 4 \\
\hline$[221]$ & $u_{1} v_{2}-u_{2} v_{1}=v_{1}^{2}$ & 4 \\
& $u_{1} v_{3}-u_{3} v_{1}=v_{1} v_{3}$ & \\
\hline$[311]$ & $u_{2}=v_{1} v_{2}$ & 4 \\
& $u_{3}=\left(1-v_{1}\right) v_{3}$ & \\
\hline$[32]$ & $u_{2}=v_{1} v_{2}$ & 5 \\
& $u_{3}=v_{2}+v_{1} v_{3}$ & \\
\hline$[41]$ & $u_{1}=v_{2}-v_{1}^{2}$ & 5 \\
& $u_{3}=\left(1-v_{1}\right) v_{3}$ & \\
\hline$[5]$ & $u_{1}=v_{2}-v_{1}^{2}$ & 6 \\
& $u_{2}=v_{3}-v_{1} v_{2}$ & \\
\hline
\end{tabular}

Systems presented in Table 1 are not SL(5)-equivalent. Even though all 4-dimensional stabilisers are commutative subalgebras of $\mathbf{S L}(5)$, they are not conjugate (in particular, only the first of them is a Cartan subalgebra).

Remark 2. Note that Table 1 does not contain a linear system. It can be recovered from the following generalisation of the Chasles construction. Take projective space $\mathbb{P}^{4}$ with homogeneous coordinates $\xi=\left(\xi^{1}: \xi^{2}: \xi^{3}: \xi^{4}: \xi^{5}\right)$, let $A$ and $B$ be two $5 \times 5$ matrices from SL(5). Consider the family of lines $\overline{(\eta, \varphi)}$ through $\eta=\xi A$ and $\varphi=\xi B$. A linear system corresponds to the case when the pencil $A+\lambda B$ consist of two Kronecker blocks of the size $1 \times 2$ and $4 \times 3$ (Kronecker blocks of other size lead to degenerate systems).

Proposition 6. The Chasles construction gives all linearly degenerate integrable systems of type (1). 


\section{Proof:}

Consider the Grassmann variety of all pencils $A+\lambda B$ in the space of $5 \times 5$ matrices. Denote by $\Sigma$ the open subset in $\mathbf{G r}(2,25)$ which corresponds to non-degenerate equations via the Chasles construction. Clearly, this is an irreducible algebraic variety. The Chasles construction defines a rational algebraic map from $\mathbf{G r}(2,25)$ to the 22-dimensional variety of all linearly degenerate integrable systems, with $\Sigma$ as the set of regular points. The fibres of this map are orbits of the right action of SL(5) on pencils $A+\lambda B$. Moreover, we can easily verify that this action has a discrete stabiliser in the case when the pencil $A+\lambda B$ is of Segre type [11111]. Therefore, generic fibres of this map are 24-dimensional. Thus, its image is an irreducible variety of dimension $2 \cdot 23-24=22$. As $\mathbf{G r}(2,25)$ is a projective variety, the image has to coincide with the set of all linearly degenerate integrable systems.

Table 2 brings together some further examples of linearly degenerate integrable systems that can be interpreted as Bäcklund transformations: on elimination of $v$, they lead to a second-order PDE for $u$, similarly, on elimination of $u$ they lead to a second-order PDE for $v$ (columns 2 and 3). Each of these systems is equivalent to one of the canonical forms presented in Table 1 (as indicated by its type).

Table 2: Examples of Bäcklund transformations

\begin{tabular}{|l|l|l|}
\hline System (1) & Equation for $u$ & Equation for $v$ \\
\hline $\begin{array}{l}\text { Type }[1111] \\
a_{1} \tilde{a}_{3} v_{t} u_{x}-a_{3} \tilde{a}_{1} v_{x} u_{t}=0 \\
a_{1} \tilde{a}_{2} v_{y} u_{x}-a_{2} \tilde{a}_{1} v_{x} u_{y}=0\end{array}$ & $a_{1} u_{x} u_{y t}+a_{2} u_{y} u_{x t}+a_{3} u_{t} u_{x y}=0$ & $\tilde{a}_{1} v_{x} v_{y t}+\tilde{a}_{2} v_{y} v_{x t}+\tilde{a}_{3} v_{t} v_{x y}=0$ \\
\hline $\begin{array}{l}\text { Type }[211] \\
(\lambda-1) v_{y}-u_{y} v_{x}=0\end{array}$ & $u_{y t}+u_{y} u_{x t}-u_{t} u_{x y}=0$ & $v_{x} v_{y t}+(\lambda-1) v_{y} v_{x t}-\lambda v_{t} v_{x y}=0$ \\
$\lambda v_{t}-u_{t} v_{x}=0$ & $u_{y y}+u_{y} u_{x t}-u_{t} u_{x y}=0$ & \\
\hline $\begin{array}{l}\text { Type }[221] \\
v_{y}-\lambda u_{y} v_{x}=0 \\
v_{t}+\left(\lambda^{2} u_{y}-\lambda u_{t}\right) v_{x}=0\end{array}$ & $u_{x y}+u_{y} u_{x t}-u_{x} u_{y t}=0$ & $v_{x t}+v_{t} v_{x y}-v_{x} v_{y t}=0$ \\
\hline $\begin{array}{l}\text { Type }[221] \\
u_{y} v_{t}-1=0 \\
u_{x}-u_{y} v_{x}=0\end{array}$ & $\left.u_{x t}+u_{x} u_{y y}-v_{y} v_{x y}-v_{x} v_{y y}\right)=0$ \\
\hline $\begin{array}{l}\text { Type }[311] \\
v_{t}+\left(\lambda-u_{y}\right) v_{y}=0 \\
\lambda v_{x}-u_{x} v_{y}=0\end{array}$ & $u_{x t}+u_{y y}+u_{y} u_{x x}-u_{x} u_{x y}=0$ & $\left(\frac{v_{y}}{v_{x}}\right)_{y}=\left(\frac{\lambda v_{y}-v_{t}}{v_{x}}\right)_{x}$ \\
\hline Type $[32]$ & & $v_{t} v_{x y}-v_{y} v_{x t}+\lambda\left(v_{y} v_{x y}-v_{x} v_{y y}\right)=0$ \\
$u_{y} v_{x}-1=0$ \\
$u_{t}-u_{y} v_{y}=0$
\end{tabular}

Some of the second-order PDEs from Table 2 have appeared in different contexts in [48, 43, 61, 10, 46, 3].

Remark 3. The equivalence group acts algebraically on the parameter space of linearly generate integrable systems, so we can consider the rational quotient $M_{\mathrm{reg}}^{22} / \mathbf{S L}(5)$. Since generic orbits 
of this action have dimension 20, the moduli space of linearly degenerate integrable systems is two-dimensional.

\subsection{Further examples in terms of modular forms and theta functions}

This section contains a list of more exotic integrable systems that are not expressible in elementary functions. These examples demonstrate that one should not expect any 'simple parametrisation' of integrable systems of type (1).

Example 1. Let us begin with the system

$$
u_{t}=v_{x}, \quad v_{t}=\frac{u_{y}}{v_{x}}+\frac{1}{6} \eta\left(u_{x}\right) v_{x}^{2}
$$

which appeared in [47] in the classification of integrable hydrodynamic chains. In this case the integrability conditions result in the Chazy equation for $\eta$,

$$
\eta^{\prime \prime \prime}+2 \eta \eta^{\prime \prime}-3 \eta^{2}=0,
$$

whose generic solution is known to be the Eisenstein series $E_{2}$ associated with the full modular group.

Example 2. Consider the system

$$
v_{x}+u_{x} u_{y} r\left(u_{t}\right)=0, \quad v_{t}=u_{y} .
$$

In this case the integrability conditions result in the third-order ODE for $r$,

$$
r^{\prime \prime \prime}\left(r^{\prime}-r^{2}\right)-r^{\prime \prime 2}+4 r^{3} r^{\prime \prime}+2 r^{\prime 3}-6 r^{2}{r^{\prime}}^{2}=0,
$$

which appeared recently in different context in the theory of modular forms of level two: compare with equation (4.7) from [1]. Its generic solution is given by the Eisenstein series

$$
r\left(u_{t}\right)=1-8 \sum_{n=1}^{\infty} \frac{(-1)^{n} n e^{4 n u_{t}}}{1-e^{4 n u_{t}}},
$$

associated with the congruence subgroup $\Gamma_{0}(2)$ of the modular group.

Example 3. As a generalisation of Example 2, let us consider the system

$$
v_{x}+u_{x} r\left(u_{y}, u_{t}\right)=0, \quad v_{t}=u_{y} .
$$

In a somewhat different representation, it first appeared in [10]. It was demonstrated that the integrability conditions imply $r=2 s^{\prime} / s$ where prime denotes differentiation by $u_{y}$, and $s\left(u_{y}, u_{t}\right)=\theta\left(\frac{u_{y}}{2 \pi},-\frac{u_{t}}{\pi i}\right)$ is the Jacobi theta function:

$$
\theta(z, \tau)=1+2 \sum_{n=1}^{\infty} e^{\pi i n^{2} \tau} \cos (2 \pi n z) .
$$

Example 4. Further generalisation,

$$
v_{x}+f\left(u_{x}, u_{y}, u_{t}\right)=0, \quad v_{t}=u_{y},
$$

was discussed in [26], where it was shown that the requirement of integrability implies that generic $f$ is given by the ratio of two Jacobi theta functions:

$$
f\left(u_{x}, u_{y}, u_{t}\right)=-\frac{1}{4} \ln \frac{\theta_{1}\left(u_{t}, u_{y}-u_{x}\right)}{\theta_{1}\left(u_{t}, u_{y}+u_{x}\right)} .
$$




\subsection{Integrable systems in terms of generalised hypergeometric functions}

In this section we utilise the construction of $[45,46]$ which parametrises integrable quasilinear equations in $2+1$ dimensions in terms of solutions of the generalised hypergeometric system,

$$
\begin{gathered}
\frac{\partial^{2} h}{\partial z_{i} \partial z_{j}}=\frac{s_{i}}{z_{i}-z_{j}} \frac{\partial h}{\partial z_{j}}+\frac{s_{j}}{z_{j}-z_{i}} \frac{\partial h}{\partial z_{i}}, \quad i \neq j, \\
\frac{\partial^{2} h}{\partial z_{i}^{2}}=-\left(1+\sum_{j=1}^{n+2} s_{j}\right) \frac{s_{i}}{z_{i}\left(z_{i}-1\right)} h+\frac{s_{i}}{z_{i}\left(z_{i}-1\right)} \sum_{j \neq i}^{n} \frac{z_{j}\left(z_{j}-1\right)}{z_{j}-z_{i}} \cdot \frac{\partial h}{\partial z_{j}}+ \\
\left(\sum_{j \neq i}^{n} \frac{s_{j}}{z_{i}-z_{j}}+\frac{s_{i}+s_{n+1}}{z_{i}}+\frac{s_{i}+s_{n+2}}{z_{i}-1}\right) \frac{\partial h}{\partial z_{i}},
\end{gathered}
$$

here $h$ is a function of $n$ variables $z_{1}, \ldots, z_{n}$, and $s_{1}, \ldots, s_{n+2}$ are arbitrary constants. This system is involutive and possesses $n+1$ linearly independent solutions known as generalised hypergeometric functions [30, 45]. Here we consider the case $n=4$. Let $\left\{h^{1}, h^{2}, g^{1}, g^{2}, g^{3}\right\}$ be a basis of solutions of system (34). Let us introduce the parametric formulae

$$
\begin{array}{ll}
u_{x}=\frac{W\left(g^{1}, h^{1}\right)}{W\left(h^{1}, h^{2}\right)}, & u_{y}=\frac{W\left(g^{2}, h^{1}\right)}{W\left(h^{1}, h^{2}\right)},
\end{array}
$$

where $W$ is the Wronskian, $W(f, g)=f g^{\prime}-g f^{\prime}$, and prime denotes differentiation with respect to one of the variables $z_{i}$ (for definiteness, we assume ${ }^{\prime}=\partial / \partial z_{4}$ ). Formulae (35) can be viewed as parametric equations of a fourfold $X \subset \mathbf{G r}(3,5)$. Eliminating the parameters $z_{1}, \ldots, z_{4}$ we obtain system of type (1).

Proposition 7. Parametric equations (35) define generic integrable system of type (1).

\section{Proof:}

Given hypergeometric system (34), the construction of [45] requires an additional ingredient, namely, the choice of a $k$-dimensional subspace of solutions of system (34). We will only need a particular case of the general scheme that corresponds to $n=4, k=2$. Thus, let $h^{1}, h^{2}$ be a pair of linearly independent solutions of system (34). Let us complete this pair to a basis $\left\{h^{1}, h^{2}, g^{1}, g^{2}, g^{3}\right\}$, and introduce the following system of 6 first-order quasilinear PDEs for $z_{1}, \ldots, z_{4}$, considered as functions of the auxiliary variables $x, y, t$ :

$$
\begin{aligned}
& \left(\frac{W\left(g^{1}, h^{1}\right)}{W\left(h^{1}, h^{2}\right)}\right)_{y}=\left(\frac{W\left(g^{2}, h^{1}\right)}{W\left(h^{1}, h^{2}\right)}\right)_{x}, \quad\left(\frac{W\left(g^{1}, h^{1}\right)}{W\left(h^{1}, h^{2}\right)}\right)_{t}=\left(\frac{W\left(g^{3}, h^{1}\right)}{W\left(h^{1}, h^{2}\right)}\right)_{x}, \quad\left(\frac{W\left(g^{2}, h^{1}\right)}{W\left(h^{1}, h^{2}\right)}\right)_{t}=\left(\frac{W\left(g^{3}, h^{1}\right)}{W\left(h^{1}, h^{2}\right)}\right)_{y}, \\
& \left(\frac{W\left(g^{1}, h^{2}\right)}{W\left(h^{1}, h^{2}\right)}\right)_{y}=\left(\frac{W\left(g^{2}, h^{2}\right)}{W\left(h^{1}, h^{2}\right)}\right)_{x}, \quad\left(\frac{W\left(g^{1}, h^{2}\right)}{W\left(h^{1}, h^{2}\right)}\right)_{t}=\left(\frac{W\left(g^{3}, h^{2}\right)}{W\left(h^{1}, h^{2}\right)}\right)_{x}, \quad\left(\frac{W\left(g^{2}, h^{2}\right)}{W\left(h^{1}, h^{2}\right)}\right)_{t}=\left(\frac{W\left(g^{3}, h^{2}\right)}{W\left(h^{1}, h^{2}\right)}\right)_{y} .
\end{aligned}
$$

It was demonstrated in [45] that this system is integrable by the method of hydrodynamic reductions, and possesses a dispersionless Lax pair. The conservative structure of this system implies the existence of potentials $u$ and $v$ specified by (35). Thus, parametric equations (35) indeed give rise to integrable system (1). Since the moduli space of integrable systems (1) is 6-dimensional 
and, for $n=4$, hypergeometric system (34) depends on 6 essential parameters $s_{1}, \ldots, s_{6}$, the claim follows. One can show that transformations from the equivalence group $\mathbf{S L}(5)$ are in one-to-one correspondence with linear transformations of the basis $\left\{h^{1}, h^{2}, g^{1}, g^{2}, g^{3}\right\}$.

We emphasise that, although formulae (35) parametrise generic integrable systems (1), the degeneration procedure leading to examples from Section 4.3 is far from trivial.

\section{Geometric aspects of integrability}

In this section we adopt a geometric point of view and consider system (1) as equations defining a fourfold $X$ in the Grassmannian $\mathbf{G r}(3,5)$. Our aim is to reformulate the integrability conditions in intrinsic geometric terms.

\section{1 $\mathbf{G L}(2, \mathbb{R})$ structure on a fourfold $X \subset \mathbf{G r}(3,5)$}

Let $X$ be a non-degenerate fourfold in the Grassmannian $\mathbf{G r}(3,5)$. Taking a point $o \in X$ and projectivising the intersection of the tangent space $\mathrm{T}_{o} X$ with the Segre cone $C$ in $\mathrm{T}_{o} \mathbf{G r}(3,5)$ one obtains a twisted cubic, that is, a rational normal curve of degree three. This twisted cubic can also be interpreted as the set of matrices of rank one in the tangent space $\mathrm{T}_{o} X$ when we identify $\mathrm{T}_{o} \mathbf{G r}(3,5)$ with the space of $2 \times 3$ matrices. Thus, the projectivised tangent bundle of the fourfold $X$ is equipped with a field of twisted cubics. This supplies $X$ with a $\mathbf{G L}(2, \mathbb{R})$ structure.

Alternatively, one can say that each tangent space to $X$ is identified with a four-dimensional space of binary cubics: in this picture the rational normal curve corresponds to cubics with a triple root. In representation-theoretic language, the tangent space $T_{o} X$ is an irreducible $\mathfrak{s l}(2)$ module $V_{3}$; here and below $V_{l} \simeq S^{l}\left(\mathbb{R}^{2}\right)$ is the irreducible $\mathfrak{s l}(2)$-representation of dimension $l+1$.

Remark. Another natural source of $\mathbf{G L}(2, \mathbb{R})$ structures in four dimensions is provided by 4-th order ODEs with vanishing Wünschmann invariants $[8,15,21,44]$. In this context, $\mathbf{G L}(2, \mathbb{R})$ structures are induced on the parameter spaces of solutions to the corresponding ODEs. We emphasise that the structures coming from ODEs always have zero torsion (so that all geometry is contained in the curvature), while in our case the torsion is generally nonzero, and defines the curvature.

The most important property of $\mathbf{G L}(2, \mathbb{R})$ structures in four dimensions is the existence of a unique intrinsic connection (the Bryant connection) whose torsion takes values in the 8dimensional irreducible representation of $\mathbf{G L}(2, \mathbb{R})[8]$. Let us recall the construction. The cubic cones of the $\mathbf{G L}(2, \mathbb{R})$ structure induced on $X$ are defined as the intersection of 3 quadrics, $\omega^{\alpha}=0$, where

$$
\omega^{1}=d a d q-d b d p, \quad \omega^{2}=d a d g-d p d f, \quad \omega^{3}=d b d g-d q d f ;
$$

here $f(a, b, p, q)$ and $g(a, b, p, q)$ correspond to the right-hand sides of system (8). The tangent space $T_{o} X$ is an irreducible $\mathfrak{s l}(2)$ module $V_{3}$, and since $\Lambda^{2} V_{3}=V_{0}+V_{4}$, it has a canonical conformal almost symplectic structure $\Omega$ (see Sect. 5.3 for explicit formulae). Raising one index of $\omega^{\alpha}$ by means of $\Omega$ we obtain 3 operators $A^{\alpha} \in \mathfrak{g l}(4), \alpha=1,2,3$, that form a basis of the irreducible embedding of $\mathfrak{s l}(2)$ into $\mathfrak{g l}(4)$. Define the bilinear form

$$
B^{\alpha \beta}=\operatorname{tr} A^{\alpha} A^{\beta},
$$


which, up to a constant factor, is the Killing form of $\mathfrak{s l}(2)$. Let $B^{\#}$ be the inverse of $B$, and let

$$
C=20 B_{\alpha \beta}^{\#} A^{\alpha} A^{\beta}
$$

be the (normalized) Casimir operator. Then $C$ is a well-defined element of the universal enveloping algebra of $\mathfrak{s l}(2)$ which is independent of the choice of the basis $\omega^{\alpha}$ (the coefficient 20 appears here because we use the embedding of $\mathfrak{s l}(2)$ into $\mathfrak{g l}(4)$, rather than the adjoint representation, to define the Casimir operator). The Casimir $C$ acts naturally on any irreducible $\mathfrak{s l}(2)$ module $V_{l}$ as a scalar operator (due to the Schur lemma),

$$
C_{V_{l}}=\lambda_{l} \mathrm{Id}, \quad \lambda_{l}=l(l+2) .
$$

The Bryant connection $\nabla$ is uniquely defined by the following two properties [8]:

- $\nabla \omega^{\alpha}=0 \bmod \left\langle\omega^{\beta}\right\rangle$. This means that $\nabla$ preserves the $\mathbf{G L}(2, \mathbb{R})$ structure.

- The torsion $T$ of the Bryant connection $\nabla$ lies in the unique 8-dimensional submodule $V_{7}$ of the $\mathfrak{s l}(2)$-representation in the space of all algebraic torsions. This can be expressed via the following linear condition:

$$
C \cdot T=\lambda_{7} T=63 T \text {. }
$$

In more detail, the first condition gives 56 equations for the 64 Christoffel's symbols $\Gamma_{j k}^{i}$ of the connection $\nabla$. Of these 48 are linearly independent, so that $\nabla$ exists with the freedom of $64-48=16$ arbitrary functions. To fix these functions we note that since the tangent space $\tau=T_{o} X$ is an irreducible $\mathfrak{s l}(2)$ module $V_{3}$, the torsion $T$ of $\nabla$ decomposes as follows:

$$
\Lambda^{2} \tau^{*} \otimes \tau=\left(V_{0} \oplus V_{4}\right) \otimes V_{3}=V_{1} \oplus 2 V_{3} \oplus V_{5} \oplus V_{7} .
$$

We can change the connection by a $\mathfrak{g l}(2)$-equivariant gauge from $\operatorname{Hom}(\tau, \mathfrak{g l}(2))$, which decomposes as $V_{3} \otimes\left(V_{0}+V_{2}\right)=V_{1} \oplus 2 V_{3} \oplus V_{5}$. This removes the freedom of 16 arbitrary functions and yields a unique connection with torsion $T \in V_{7}$. Note that the action of the operators $A^{\alpha}$ on tensors is standard, for instance, for the torsion tensor one has $\left(A^{\alpha} \cdot T\right)_{i j}^{k}=$ $\left(A^{\alpha}\right)_{a}^{k} T_{i j}^{a}-\left(A^{\alpha}\right)_{i}^{a} T_{a j}^{k}-\left(A^{\alpha}\right)_{j}^{a} T_{i a}^{k}$. This defines the action of the universal enveloping algebra, in particular, of the Casimir operator $C$.

\subsection{Geometric interpretation of the integrability conditions}

As explained in Sect. 5.1, each projectivised tangent space $\mathrm{T}_{o} X$ carries a rational normal curve $\gamma$ of degree three.

\section{Definition.}

(a) Bisecant surface is a two-dimensional submanifold $\Sigma^{2} \subset X$ whose projectivised tangent planes are bisecant lines of $\gamma$.

(b) Given a three-dimensional submanifold $\Sigma^{3} \subset X$, each tangent space $\mathrm{T}_{o} \Sigma^{3}$ carries three distinguished directions, namely those corresponding to the three points of intersection of $\mathbb{P T}_{o} \Sigma^{3}$ with $\gamma$. These directions define a net on $\Sigma^{3}$, we will call it the characteristic net. Holonomic submanifolds $\Sigma^{3}$ are defined by the requirement that the characteristic net is holonomic (that is, locally a coordinate net).

Proposition 8. Bisecant surfaces and holonomic submanifolds $\Sigma^{3}$ correspond to two- and threecomponent hydrodynamic reductions of system (1). Furthermore, 
(i) every fourfold $X$ possesses infinitely many bisecant surfaces parametrised by two arbitrary functions of one variable;

(ii) a fourfold $X$ corresponds to an integrable system if and only if it possesses infinitely many holonomic submanifolds $\Sigma^{3}$ parametrised by three arbitrary functions of one variable. Thus, the existence of holonomic submanifolds $\Sigma^{3}$ is a geometric characterisation of integrability.

\section{Proof:}

We follow the notation of Sect. 2.1. Let us represent our system in quasilinear form (9), and take an $N$-component reduction specified by equations (10). The geometric image in the Grassmannian $\mathbf{G r}(3,5)$ is a submanifold $\Sigma^{N} \subset X$ (it would be sufficient for our purposes to restrict to $N=2,3$ ), represented by the symmetric matrix

$$
U=\left(\begin{array}{lll}
a & b & f \\
p & q & g
\end{array}\right)
$$

parametrised by $R^{1}, \ldots, R^{N}$. Using equations (11), (12) one obtains

$$
\partial_{i} U=\left(\begin{array}{ccc}
\partial_{i} a & \mu^{i} \partial_{i} a & \lambda^{i} \partial_{i} a \\
\partial_{i} p & \mu^{i} \partial_{i} p & \lambda^{i} \partial_{i} p
\end{array}\right) .
$$

Thus, $\partial_{i} U$ is a matrix of rank one, so that the projectivisation of $\partial_{i} U$ belongs to the rational normal curve $\gamma$, and coordinates $R^{i}$ provide the characteristic net on $\Sigma^{N}$. For $N=2$ we have a two-dimensional surface $\Sigma^{2} \subset X$ parametrised by $R^{1}, R^{2}$. Since both $\partial_{1} U$ and $\partial_{2} U$ have rank one, the surface $\Sigma^{2}$ is bisecant. Since every system of type (1) (not necessarily integrable) possesses infinitely many two-component reductions parametrised by two arbitrary functions of one variable, every fourfold $X$ of the Grassmannian $\mathbf{G r}(3,5)$ possesses infinitely many bisecant surfaces. This establishes part (i) of the Proposition.

Similarly, every three-component reduction corresponds to a holonomic submanifold $\Sigma^{3} \subset$ $X$ parametrised by $R^{1}, R^{2}, R^{3}$. Since an integrable system possesses (by definition) infinitely many three-component reductions parametrised by three arbitrary functions of one variable, the corresponding fourfold $X$ possesses infinitely many holonomic submanifolds $\Sigma^{3}$. This establishes the first part of (ii).

To finish the proof one needs to show that, conversely, bisecant surfaces (holonomic submanifolds $\Sigma^{3}$ ) of $X$ correspond to two-component (three-component) reductions of the associated system. This can be demonstrated as follows. Let $\Sigma^{2}$ be a bisecant surface represented in form (36), referred to its characteristic net $R^{1}, R^{2}$. Thus, $a, b, p, q, f, g$ are functions of $R^{1}, R^{2}$ such that the rank of $\partial_{i} U$ equals one, so that one can introduce parametrisation (37). Compatibility conditions for the equations $\partial_{i} b=\mu^{i} \partial_{i} a$ and $\partial_{i} q=\mu^{i} \partial_{i} p$ imply

$$
\partial_{i} \partial_{j} a=\frac{\partial_{j} \mu^{i}}{\mu^{j}-\mu^{i}} \partial_{i} a+\frac{\partial_{i} \mu^{j}}{\mu^{i}-\mu^{j}} \partial_{j} a \quad \text { and } \quad \partial_{i} \partial_{j} p=\frac{\partial_{j} \mu^{i}}{\mu^{j}-\mu^{i}} \partial_{i} p+\frac{\partial_{i} \mu^{j}}{\mu^{i}-\mu^{j}} \partial_{j} p,
$$

respectively. Similarly, compatibility conditions for the equations $\partial_{i} f=\lambda^{i} \partial_{i} a$ and $\partial_{i} g=\lambda^{i} \partial_{i} p$ imply

$$
\partial_{i} \partial_{j} a=\frac{\partial_{j} \lambda^{i}}{\lambda^{j}-\lambda^{i}} \partial_{i} a+\frac{\partial_{i} \lambda^{j}}{\lambda^{i}-\lambda^{j}} \partial_{j} a \quad \text { and } \quad \partial_{i} \partial_{j} p=\frac{\partial_{j} \lambda^{i}}{\lambda^{j}-\lambda^{i}} \partial_{i} p+\frac{\partial_{i} \lambda^{j}}{\lambda^{i}-\lambda^{j}} \partial_{j} p .
$$

Subtracting these equations from each other we get

$$
s_{i j} \partial_{i} a+s_{j i} \partial_{j} a=0, \quad s_{i j} \partial_{i} p+s_{j i} \partial_{j} p=0,
$$


where $s_{i j}=\partial_{j} \mu^{i} /\left(\mu^{j}-\mu^{i}\right)-\partial_{j} \lambda^{i} /\left(\lambda^{j}-\lambda^{i}\right)$. The case $s_{i j}=s_{j i}=0$ gives commutativity conditions (7), so that we recover all equations governing two-component reductions. If one of the coefficients $s_{i j}$ or $s_{j i}$ is nonzero, then the functions $a$ and $p$ must be functionally dependent, so that $d p=c d a$ for some coefficient $c$. But then we must also have $d q=c d b$ and $d g=c d f$, so that the whole tangent space of the surface $\Sigma^{2}$ consists of rank one matrices. This, however, is not possible for non-degenerate systems (1).

For holonomic submanifolds $\Sigma^{3}$, considerations are essentially the same. This finishes the proof of Proposition 8.

Remark. The proof of Proposition 8 is similar in spirit to that of Theorem 3 of [27] in the context of dispersionless Hirota type equations. There is however one remarkable difference: although every Hirota type equation corresponds to a hypersurface $M^{5}$ in the Lagrangian Grassmannian $\Lambda^{6}$ so that three-component reductions correspond to holonomic trisecant submanifolds of the natural $\mathbf{G L}(2, \mathbb{R})$ structure induced on $M^{5}$, the requirement of holonomicity becomes redundant. Namely, it was shown by Smith [56] that the existence of trisecant submanifolds implies the existence of holonomic trisecant submanifolds (this is due to the fact that not every 3-plane in $T M^{5}$ is trisecant). On the contrary, for a fourfold $X \subset \mathbf{G r}(3,5)$ every 3-plane in $T X$ is trisecant, and the requirement of holonomicity is the only constraint.

\subsection{Canonical conformal almost symplectic structure}

Let $\mathbb{P}^{3}$ be a projective space with homogeneous coordinates $\left(x^{0}: x^{1}: x^{2}: x^{3}\right)$. A rational normal curve $\gamma$ of degree three has the standard form $\gamma=\left(1: t: t^{2}: t^{3}\right)$. One can verify that, up to a scalar factor, there exists a unique 2-form, $\omega=d x^{0} \wedge d x^{3}-3 d x^{1} \wedge d x^{2}$, for which all tangent planes to the cone over $\gamma$ are Lagrangian, that is, $\omega\left(\gamma, \gamma^{\prime}\right)=0$. The matrix of $\omega$ is

$$
\omega=\left(\begin{array}{cccc}
0 & 0 & 0 & 1 \\
0 & 0 & -3 & 0 \\
0 & 3 & 0 & 0 \\
-1 & 0 & 0 & 0
\end{array}\right)
$$

Since each projectivised tangent space of $X$ contains a rational normal curve, the 'curved' version of the above construction supplies $X$ with a nondegenerate 2 -form $\Omega$ defined up to a conformal factor, that is, a conformal almost symplectic structure.

The differential of $\Omega$ can be represented in the form $d \Omega=\varphi \wedge \Omega$ where $\varphi$ is the so-called Lee form of $\Omega$ (in four dimensions such representation exists, and is unique). Since the rescaling $\Omega \rightarrow f \Omega$ gives rise to the transformation $\varphi \rightarrow \varphi+d f / f$, the differential $d \varphi$ is independent of the conformal factor $f$. In particular, the condition $d \varphi=0$ is (locally) equivalent to the existence of a function $f$ such that $f \Omega$ is symplectic (such structures $\Omega$ are also known as conformally symplectic).

Proposition 9. The canonical structure $\Omega$ on the fourfold $X$ corresponding to integrable system (1) is locally conformally symplectic. If $H^{1}(X, \mathbb{R})=0$, and $X$ is non-degenerate, then there exists a symplectic structure $\Omega$ on $X$ defined canonically up to multiplication by a constant. It is parallel with respect to the Bryant connection.

\section{Proof:}

Following the notation of Sect. 2.1 we parametrise $X \subset \mathbf{G r}(3,5)$ by local coordinates $a, b, p, q$ in the form

$$
X=\left(\begin{array}{ccc}
a & b & f(a, b, p, q) \\
p & q & g(a, b, p, q)
\end{array}\right) .
$$


The field of rational normal curves $\gamma$ in the projectivised tangent space of $X$ is specified by the equations $r k(d X)=1$, that is

$$
d a d q-d b d p=0, \quad d a d g-d f d p=0, \quad d b d g-d f d q=0,
$$

or, explicitly, $\gamma=(m: n: m t: n t)$ where $m=g_{b}+\left(g_{q}-f_{b}\right) t-f_{q} t^{2}, n=-g_{a}+\left(f_{a}-g_{p}\right) t+f_{p} t^{2}$, and $t$ is a parameter. In matrix notation we have $\gamma=\left(1: t: t^{2}: t^{3}\right) \cdot A$, where

$$
A=\left(\begin{array}{cccc}
g_{b} & -g_{a} & 0 & 0 \\
g_{q}-f_{b} & f_{a}-g_{p} & g_{b} & -g_{a} \\
-f_{q} & f_{p} & g_{q}-f_{b} & f_{a}-g_{p} \\
0 & 0 & -f_{q} & f_{p}
\end{array}\right) .
$$

Thus, the matrix of the 2 -form $\Omega$ is given by $\Omega=A^{-1} \omega\left(A^{-1}\right)^{T}$. It remains to calculate the corresponding 1-form $\varphi$, and verify that the condition $d \varphi=0$ holds identically modulo integrability conditions (17) satisfied by $f$ and $g$. We point out that $\operatorname{det} \Omega=9 /(\operatorname{det} A)^{2}$, furthermore, the condition $\operatorname{det} A=0$ is equivalent to the reducibility of dispersion relation (13). Thus, our assumption of non-degeneracy of the characteristic variety is equivalent to non-degeneracy of $\Omega$. Finally, one can verify by direct calculation that the associated symplectic structure, which is unique up to a constant factor, is parallel in the Bryant connection: this follows from the fact that both the connection and the symplectic structure are defined in a canonical way. This finishes the proof of Proposition 9.

We emphasize that the condition $d \varphi=0$ is necessary, but not generally sufficient for integrability. For instance, one can show that this condition is satisfied for every system of the form $u_{t}=f\left(u_{x}\right)+v_{y}, u_{y}=v_{x}$, where the function $f$ is arbitrary, whereas the integrability implies $f^{\prime \prime \prime}=0$. On the other hand, for systems of the form $u_{t}=v_{y}, v_{t}=u_{y}+g\left(u_{x}\right)$, the condition $d \varphi=0$ implies $g^{\prime \prime \prime} g^{\prime}-2 g^{\prime \prime 2}=0$, which is precisely the integrability condition. We skip details of these calculations.

\subsection{Integrability conditions via differential invariants}

Let $\nabla$ be the Bryant connection of a $\mathbf{G L}(2, \mathbb{R})$-structure on $X$. Its basic invariant is the torsion $T \in V_{7}$. Other tensorial invariants include the curvature $R$ and the covariant derivative of torsion, $\nabla T$. Let us give here explicit coordinate formulae that show our conventions (for nonsymmetric connections):

$$
\begin{gathered}
\nabla_{k} \partial_{i}=\Gamma_{i k}^{a} \partial_{a}, \quad \nabla_{k} d x^{i}=-\Gamma_{a k}^{i} d x^{a}, T_{i j}^{k}=\left\langle\nabla_{i} \partial_{j}-\nabla_{j} \partial_{i}, d x^{k}\right\rangle=\Gamma_{j i}^{k}-\Gamma_{i j}^{k}, \\
R_{l i j}^{k}=\left\langle\left[\nabla_{i}, \nabla_{j}\right] \partial_{l}, d x^{k}\right\rangle=\partial_{i} \Gamma_{l j}^{k}-\partial_{j} \Gamma_{l i}^{k}+\Gamma_{l j}^{a} \Gamma_{a i}^{k}-\Gamma_{l i}^{a} \Gamma_{a j}^{k} \\
(\nabla T)_{l i j}^{k}=\partial_{l} T_{i j}^{k}+\Gamma_{a l}^{k} T_{i j}^{a}-\Gamma_{i l}^{a} T_{a j}^{k}-\Gamma_{j l}^{a} T_{i a}^{k}
\end{gathered}
$$

Notice that both $R$ and $\nabla T$ (which are third-order differential invariants of the corresponding system (8) as $\Gamma_{i j}^{k}$ are second-order quantities in partial derivatives of $f$ and $g$ ), belong to the space $\tau \otimes \tau^{*} \otimes \Lambda^{2} \tau^{*}$ which has the following $\mathfrak{s l}(2)$ decomposition into irreducibles (with multiplicities):

$$
\tau \otimes \tau^{*} \otimes \Lambda^{2} \tau^{*}=2 V_{0} \oplus 4 V_{2} \oplus 5 V_{4} \oplus 4 V_{6} \oplus 2 V_{8} \oplus V_{10} .
$$

Consequently, we can decompose the basic third-order invariants as follows:

$$
R=R_{(0)}+R_{(2)}+R_{(4)}+R_{(6)}, \quad \nabla T=\nabla T_{(4)}+\nabla T_{(6)}+\nabla T_{(8)}+\nabla T_{(10)},
$$


where the subscript $(k)$ indicates the weight, or equivalently the (multiple) submodule $V_{k}$ to which the component belongs. Indeed, the spaces where these tensors take values decompose into $\mathfrak{s l}(2)$-irreducibles as follows:

$$
\mathfrak{g l}(2) \otimes \Lambda^{2} \tau^{*}=V_{0} \oplus 2 V_{2} \oplus 2 V_{4} \oplus V_{6}, \quad \tau^{*} \otimes V_{7}=V_{4} \oplus V_{6} \oplus V_{8} \oplus V_{10} .
$$

For a tensor $K$, the condition $K \in V_{l}$ is equivalent to $C \cdot K=l(l+2) K$. This gives a decomposition of $R$ and $\nabla T$ into eigenspaces of $C$.

By Proposition 5 of Sect. 4.2, system (1) is linearly degenerate and integrable if and only if the associated Bryant connection is trivial: $T=R=0$ (this is also a consequence of Theorem 3 below). It turns out that in the general, not linearly degenerate case, the integrability is characterised by the condition that both $R$ and $\nabla T$ can be represented as certain quadratic expressions in the torsion $T$. To write down these expressions we introduce the following tensors $T^{2}, T_{\alpha}^{2}, T_{\beta}^{2}, T_{\gamma}^{2}, T_{\delta}^{2} \in \tau \otimes \tau^{*} \otimes \Lambda^{2} \tau^{*}$ (the upper index 2 indicates that these tensors are quadratic in $T$, and $\alpha, \beta, \gamma, \delta$ are labels, not indices):

$$
\begin{gathered}
\left(T^{2}\right)_{l i j}^{k}=T_{l a}^{k} T_{i j}^{a}, \quad\left(T_{\alpha}^{2}\right)_{l i j}^{k}=T_{l a}^{k} \Omega^{a b} T_{b[i}^{c} \Omega_{c j]}, \quad\left(T_{\beta}^{2}\right)_{l i j}^{k}=T_{[j a}^{k} \Omega^{a b} T_{b l}^{c} \Omega_{c i]}, \\
\left(T_{\gamma}^{2}\right)_{l i j}^{k}=T_{[i a}^{k} \Omega^{a b} T_{b j]}^{c} \Omega_{c l}, \quad\left(T_{\delta}^{2}\right)_{l i j}^{k}=\Omega^{k a} T_{a l}^{b} \Omega_{b c} T_{i j}^{c} ;
\end{gathered}
$$

here square brackets denote skew-symmetrization in $i, j$, and $\Omega$ is the canonical almost symplectic structure (note that these tensors are independent of the choice of a conformal factor of $\Omega$ ). Due to the identity $T^{2}=2 T_{\alpha}^{2}$, the above formulae give 4 essentially different invariant tensors that are quadratic in the second-order partial derivatives of $f$ and $g$. For every $\sigma \in\{\alpha, \beta, \gamma, \delta\}$ we decompose $T_{\sigma}^{2}$ into $\mathfrak{s l}(2)$-irreducibles, where apriori all weights $0 \leq l \leq 10, l \in 2 \mathbb{Z}$, are possible:

$$
T_{\sigma}^{2}=T_{\sigma(0)}^{2}+T_{\sigma(2)}^{2}+T_{\sigma(4)}^{2}+T_{\sigma(6)}^{2}+T_{\sigma(8)}^{2}+T_{\sigma(10)}^{2} .
$$

We claim that in fact

$$
T_{\sigma(0)}^{2}=T_{\sigma(4)}^{2}=T_{\sigma(8)}^{2}=0 \text { for all } \sigma \in\{\alpha, \beta, \gamma, \delta\} .
$$

Indeed, these tensors are contractions of $T \otimes T \in S^{2} V_{7}=V_{2} \oplus V_{6} \oplus V_{10} \oplus V_{14}$, which is then projected to the right hand side of (38). Thus, $V_{14}$ disappears, and only components $V_{k}$ with $k=2,6,10$ remain in the decomposition.

Theorem 3 For non-degenerate system (1), the integrability is equivalent to the following relations among the invariants of the associated $\mathbf{G L}(2, \mathbb{R})$ structure:

$$
\begin{gathered}
R_{(0)}=0, \quad R_{(4)}=0, \quad \nabla T_{(4)}=0, \quad \nabla T_{(8)}=0, \quad \nabla T_{(10)}=-28 T_{\alpha(10)}^{2}, \\
R_{(2)}=\frac{44}{3} T_{\alpha(2)}^{2}+2 T_{\beta(2)}^{2}-\frac{40}{3} T_{\gamma(2)}^{2}-2 T_{\delta(2)}^{2}, \\
R_{(6)}=-24 T_{\alpha(6)}^{2}-30 T_{\beta(6)}^{2}-60 T_{\gamma(6)}^{2}-24 T_{\delta(6)}^{2}, \\
\nabla T_{(6)}=-8 T_{\alpha(6)}^{2}-8 T_{\beta(6)}^{2}-16 T_{\gamma(6)}^{2}-4 T_{\delta(6)}^{2} .
\end{gathered}
$$

Note that for $k=2,6$ there are 4 linearly independent components $T_{\alpha(k)}^{2}, T_{\beta(k)}^{2}, T_{\gamma(k)}^{2}, T_{\delta(k)}^{2}$ of weight $k$, and $V_{k}$ has multiplicity 4 in the $\mathfrak{s l}(2)$-submodule $\Pi_{(k)}=\left(\tau \otimes \tau^{*} \otimes \Lambda^{2} \tau^{*}\right)_{(k)}$. Thus, it is expected that $R_{(k)}$ and $\nabla T_{(k)}$ decompose in this 'basis' (since the integrability conditions reduce third-order expressions to second-order). Similarly, for $k=10$ just one $T_{\alpha(10)}^{2}$ is a basis of $\Pi_{(10)}$, and $\nabla T_{(10)}$ is expressed through it. However, the tensors $T_{\sigma}^{2}$ have no $V_{k}$-components for $k=0,4,8$, and for the corresponding third-order tensors we get simpler relations. 


\section{Proof:}

The proof is computational. To verify these formulae we substitute the integrability conditions (17). This leads to expressions that are quadratic in the second-order partial derivatives of $f$ and $g$, with coefficients depending on their first-order derivatives (that is, functions on $J^{1}$ ). The computational complexity of the output is quite high, however, the substitution of any 'generic' point of $J^{1}$ readily gives zero (this verification is done in Mathematica; we choose a rational point so that all computations are exact and rigorous). Here the choice of a generic point is irrelevant due to the transitivity of $\mathbf{S L}(5)$-action on $J^{1}$, see discussion at the end of Sect. 3.

Conversely, one has to verify that the above relations imply all of the 40 integrability conditions (17). Note that these relations are linear in third-order partial derivatives of $f$ and $g$, with coefficients being functions on $J^{1}$. The rank of the matrix at third-order derivatives is equal to 40 (one can again restrict to any generic point of $J^{1}$ ), and this implies the claim.

Remark. Some of the relations from Theorem 3 hold identically for every fourfold $X \subset \mathbf{G r}(3,5)$, without using the integrability conditions. These include the relations

$$
R_{(0)}=0, T^{2}=2 T_{\alpha}^{2}, T_{\gamma(10)}^{2}=-T_{\alpha(10)}^{2}, T_{\beta(10)}^{2}=T_{\delta(10)}^{2}=0,
$$

that play the role of obstructions to the embeddability of an abstract $\mathbf{G L}(2, \mathbb{R})$ structure into the Grassmannian $\mathbf{G r}(3,5)$. It would be interesting to find a complete set of such obstructions.

\section{Concluding remarks}

This paper is a first step towards the general theory of integrability in Grassmann geometries. We gave a detailed characterisation of integrable systems $\Sigma(X)$ associated with fourfolds $X \subset$ $\operatorname{Gr}(3,5)$.

- We believe that some of our results can be generalised as follows.

(a) In the dimension $d=3$, the parameter space of non-degenerate integrable systems $\Sigma(X)$ associated with submanifolds $X$ of codimension $n-3 \geq 2$ in $\mathbf{G r}(3, n)$ is finite-dimensional. Submanifolds corresponding to 'generic' integrable systems are not algebraic.

(b) In higher dimensions $d \geq 4$, every non-degenerate integrable system $\Sigma(X)$ associated with a submanifold of codimension $n-d \geq 2$ in $\operatorname{Gr}(d, n)$ is necessarily linearly degenerate. Submanifolds $X$ corresponding to linearly degenerate integrable systems are rational (generally, singular), see [17] for the first steps in this direction.

At the moment we are not aware of any $(n, d)$-independent approaches to the results of this kind.

- Some examples of integrable systems (1) discussed in this paper can be interpreted as Bäcklund transformations: on elimination of $v$, they lead to a second-order PDE for $u$, similarly, on elimination of $u$ they lead to a second-order PDE for $v$. It would be interesting to obtain a classification of Bäcklund transformations.

- Our definition of integrability, based on the existence of holonomic trisecant submanifolds, applies to any abstract $\mathbf{G L}(2, \mathbb{R})$ structure. It would be of interest to understand whether every integrable $\mathbf{G L}(2, \mathbb{R})$ structure is necessarily embeddable, that is, comes from a fourfold $X \subset \mathbf{G r}(3,5)$. More generally, one may ask for a criterion of embeddability of an abstract $\mathbf{G L}(2, \mathbb{R})$ structure (see Remark at the end of Sect. 5.4). 
- It is a true challenge to classify integrable systems that correspond to algebraic fourfolds $X \subset \mathbf{G r}(3,5)$. The homology class of any such $X$ can be represented as $a \sigma+b \eta$ where $a, b$ are nonnegative integers, and $\sigma, \eta$ are the standard four-dimensional Schubert cycles: $\sigma$ corresponds to 3-dimensional subspaces containing a fixed 1-dimensional subspace, and $\eta$ corresponds to 3-dimensional subspaces that have 2-dimensional intersections with a fixed 3 -dimensional subspace. Which values of $a$ and $b$ are compatible with the requirement of integrability? The approach of $[9,51]$ allows one to characterise algebraic $X$ (in some special homology classes) as integral manifolds of certain overdetermined exterior differential systems. Provided such characterisation is found for every $a$ and $b$, it would be straightforward to intersect this differential system with our integrability conditions.

\section{Acknowledgements}

We thank A Bolsinov, R Bryant, I Dolgachev, E Mezzetti, M Pavlov, A Prendergast-Smith and $\mathrm{C}$ Robles for clarifying discussions, as well as the referee for useful comments. We also thank the LMS for their support of B Doubrov and B Kruglikov to Loughborough making this collaboration possible. The research of E Ferapontov was partially supported by the EPSRC grant EP/N031369/1.

\section{References}

[1] M.J. Ablowitz, S. Chakravarty and H. Hahn, Integrable systems and modular forms of level 2, J. Phys. A: Math. Gen. 39 (2006) 15341-15353.

[2] S.I. Agafonov and E.V. Ferapontov, Systems of conservation laws in the setting of the projective theory of congruences: reducible and linearly degenerate systems, Differential Geometry and its Applications 17 (2002) 153-173.

[3] H. Baran, I.S. Krasilshchik, O.I. Morozov and P. Vojcak, Symmetry reductions and exact solutions of Lax integrable 3-dimensional systems, arXiv:1407.0246.

[4] W. Bertram, Un theoréme de Liouville pour les algébres de Jordan, Bull. Soc. Math. France 124, no. 2 (1996) 299-327.

[5] L.V. Bogdanov and B.G. Konopelchenko, Nonlinear Beltrami equations and $\tau$-functions for dispersionless hierarchies, Phys. Letters A: 322, no. 5-6 (2004) 330-337.

[6] G. Boillat, Sur la forme générale du système de Monge-Ampère, C. R. Acad. Sci. Paris Sér. I Math. 325, no. 3 (1997) 339-342.

[7] C.P. Boyer and J.D. Finley, Killing vectors in self-dual Euclidean Einstein spaces, J. Math. Phys. 23 (1982) 1126-1130.

[8] R.L. Bryant, Two exotic holonomies in dimension four, path geometries, and twistor theory, in: Complex geometry and Lie theory (Sundance, UT, 1989), 33-88, Proc. Sympos. Pure Math., 53, Amer. Math. Soc., Providence, RI (1991).

[9] R.L. Bryant, Rigidity and quasi-rigidity of extremal cycles in Hermitian symmetric spaces, Annals of Mathematical Studies 153, Princeton University Press, arXiv:math/0006186 (2010).

[10] P.A. Burovskii, E.V. Ferapontov and S.P. Tsarev, Second order quasilinear PDEs and conformal structures in projective space, International J. Math. 21, no. 6 (2010) 799-841.

[11] D.M.J. Calderbank, Integrable background geometries, SIGMA 10, 034 (2014) 51pp; see also http://people.bath.ac.uk/dmjc20/mpapers.html (2002).

[12] E. Cartan, Sur une classe d'espaces de Weyl, Ann. Sci. École Norm. Sup. (3) 60 (1943) 1-16, MR0014292, Zbl 0028.30802.

[13] M. Chasles, Considérations sur la méthode générale exposée dans la séance du 15 Février, Comptes Rendus 58 (1864) 1167-76. 
[14] I.V. Dolgachev, Classical algebraic geometry. A modern view, Cambridge University Press, Cambridge, 2012, $639 \mathrm{pp}$.

[15] B. Doubrov, Contact trivialization of ordinary differential equations, Differential geometry and its applications (Opava, 2001), 73-84, Math. Publ., 3, Silesian Univ. Opava, Opava, 2001.

[16] B. Doubrov and E.V. Ferapontov, On the integrability of symplectic Monge-Ampère equations, J. Geom. Phys. 60 (2010) 1604-1616.

[17] B. Doubrov, E.V. Ferapontov, B. Kruglikov, V.S. Novikov, Integrable systems in four dimensions associated with sixfolds in $\operatorname{Gr}(4,6)$, IMRN (2018) DOI:10.1093/imrn/rnx308; arXiv:1705.06999.

[18] B.A. Dubrovin and S.P. Novikov, Hydrodynamics of weakly deformed soliton lattices. Differential geometry and Hamiltonian theory, Russian Math. Surveys 44, no. 6 (1989) 35-124.

[19] M. Dunajski, L.J. Mason and P. Tod, Einstein-Weyl geometry, the dKP equation and twistor theory, J. Geom. Phys. 37, no. 1-2 (2001) 63-93.

[20] M. Dunajski, A class of Einstein-Weyl spaces associated to an integrable system of hydrodynamic type, J. Geom. Phys. 51, no. 1 (2004) 126-137, MR2078688, Zbl 1110.53032.

[21] M. Dunajski and P. Tod, Paraconformal geometry of $n$ th-order ODEs, and exotic holonomy in dimension four, J. Geom. Phys. 56 (2006) 1790-1809.

[22] M. Dunajski and W. Krynski, Einstein-Weyl geometry, dispersionless Hirota equation and Veronese webs, Math. Proc. Cambridge Philos. Soc. 157, no. 1 (2014) 139-150.

[23] M. Dunajski, E.V. Ferapontov and B.S. Kruglikov, On the Einstein-Weyl and conformal self-duality equations, J. Math. Phys. 56, 083501 (2015); doi: 10.1063/1.4927251.

[24] E.V. Ferapontov and K.R. Khusnutdinova, On the integrability of (2+1)-dimensional quasilinear systems, Comm. Math. Phys. 248 (2004) 187-206.

[25] E.V. Ferapontov and K.R. Khusnutdinova, The characterization of two-component (2+1)-dimensional integrable systems of hydrodynamic type, J. Phys. A: Math. Gen. 37 (2004) 2949-2963.

[26] E.V. Ferapontov, K.R. Khusnutdinova and M.V. Pavlov, Classification of integrable (2+1)-dimensional quasilinear hierarchies, Theor. Math. Phys. 144 (2005) 35-43.

[27] E.V. Ferapontov, L. Hadjikos and K.R. Khusnutdinova, Integrable equations of the dispersionless Hirota type and hypersurfaces in the Lagrangian Grassmannian, International Mathematics Research Notices, (2010) 496-535; doi:10.1093/imrn/rnp134.

[28] E. V. Ferapontov and B.S. Kruglikov, Dispersionless integrable systems in 3D and Einstein-Weyl geometry, J. Diff. Geom. 97 (2014) 215-254.

[29] M.A. Gauger, On the classification of metabelian Lie algebras, Trans. Amer. Math. Soc. 179 (1973) 293-329.

[30] I.M. Gelfand, M.I. Graev, V.S. Retakh, General hypergeometric systems of equations and series of hypergeometric type, Russian Math. Surveys 47, no. 4 (1992) 1-88.

[31] J. Gibbons and S.P. Tsarev, Reductions of the Benney equations, Phys. Lett. A 211 (1996) 19-24; J. Gibbons and S.P. Tsarev, Conformal maps and reductions of the Benney equations, Phys. Lett. A 258 (1999) 263-270.

[32] S. Gindikin, Fubini-Study structures on Grassmannians, Boll. Un. Mat. Ital. B (7) 11, no. 2 (1997) 145-156.

[33] S. Gindikin and S. Kaneyuki, On the automorphism group of the generalized conformal structure of a symmetric $R$-space, Differential Geometry and its Applications 8 (1998) 21-33.

[34] A.B. Goncharov, Generalized conformal structures on manifolds, Selected translations. Selecta Math. Soviet. 6, no. 4 (1987) 307340.

[35] P. Griffiths and J. Harris, Algebraic geometry and local differential geometry, Ann. Sci. École Norm. Sup. (4) 12, no. 3 (1979) 355-452.

[36] R. Harvey and H.B. Lawson, Calibrated geometries, Acta Math. 148 (1982) 47-157.

[37] N.J. Hitchin, Complex manifolds and Einstein's equations, Twistor geometry and nonlinear systems (Primorsko, 1980), 73-99, Lecture Notes in Math. 970, Springer, Berlin-New York (1982).

[38] P.E. Jones and K.P. Tod, Minitwistor spaces and Einstein-Weyl spaces, Class. Quantum Grav. 2, no. 4 (1985) 565-577. 
[39] I.M. Krichever, The $\tau$-function of the universal Whitham hierarchy, matrix models and topological field theories, Comm. Pure Appl. Math. 47, no. 4 (1994) 437-475.

[40] S.V. Manakov and P.M. Santini, The Cauchy problem on the plane for the dispersionless KadomtsevPetviashvili equation, JETP Lett. 83 (2006) 462-6.

[41] S.V. Manakov and P.M. Santini, Inverse scattering problem for vector fields and the Cauchy problem for the heavenly equation, Phys. Lett. A 359, no 6 (2006) 613-619.

[42] S.V. Manakov and P.M. Santini, On the solutions of the second heavenly and Pavlov equations, J. Phys. A 42, no. 40 (2009) 404013, 11 pp.

[43] L. Martinez Alonso and A.B. Shabat, Towards a theory of differential constraints of a hydrodynamic hierarchy, J. Nonlinear Math. Phys. 10, no. 2 (2003) 229-242.

[44] P. Nurowski, Comment on GL(2, R) geometry of fourth-order ODEs, J. Geom. Phys. 59, no 3 (2009) 267-278.

[45] A.V. Odesskii and V.V. Sokolov, Integrable pseudopotentials related to generalized hypergeometric functions, Selecta Math. 16 (2010) 145.

[46] A.V. Odesskii and V.V. Sokolov, Integrable (2+1)-dimensional systems of hydrodynamic type, Theor. Math. Phys. 163, no. 2 (2010) 549-586.

[47] M.V. Pavlov, New integrable (2+1)-equations of hydrodynamic type, Russian Math. Surv., 58, no. 2 (2003) 384-385; M.V. Pavlov, Classifying integrable Egoroff hydrodynamic chains, Theor. Math. Phys. 138 (2004) 45-58.

[48] M.V. Pavlov, Integrable hydrodynamic chains, J. Math. Phys. 44, no. 9 (2003) 4134-4156.

[49] R. Penrose, Nonlinear gravitons and curved twistor theory, General Relativity and Gravitation 7, no. 1 (1976) 31-52.

[50] J.F. Plebański, Some solutions of complex Einstein equations, J. Math. Phys. 16 (1975) 2395-2402.

[51] C. Robles and D. The, Rigid Schubert varieties in compact Hermitian symmetric spaces, Selecta Math. (N.S.) 18, no. 3 (2012) 717-777.

[52] M. Rosenlicht, Some basic theorems on algebraic groups, Amer. J. Math. 78 (1956) 401-443.

[53] B.L. Rozdestvenskii and A.D. Sidorenko, On the impossibility of 'gradient catastrophe' for weakly nonlinear systems. Z. Vycisl. Mat. i Mat. Fiz. 7 (1967) 1176-1179.

[54] B. Sévennec, Géométrie des systèmes hyperboliques de lois de conservation, Mémoire (nouvelle série), Supplément au Bulletin de la Société Mathématique de France, 56 (1994) 1-125.

[55] J.C. Sierra, L. Ugaglia, On double Veronese embeddings in the Grassmannian G(1,N), Math. Nachr. 279, no. 7 (2006) 798-804.

[56] A.D. Smith, Integrable GL(2) Geometry and Hydrodynamic Partial Differential Equations, Comm. Anal. Geom. 18, no. 4 (2010) 743-790.

[57] A.D. Smith, A geometry for second-order PDEs and their integrability, Part 1, arXiv:1010.6010v1.

[58] S.P. Tsarev, Poisson brackets and one-dimensional Hamiltonian systems of hydrodynamic type, Soviet Math. Dokl. 31 (1985) 488-491; S.P. Tsarev, The geometry of Hamiltonian systems of hydrodynamic type. The generalized hodograph method, Math. USSR Izvestiya 37 (1991) 397-419.

[59] R.S. Ward, Einstein-Weyl spaces and $S U(\infty)$ Toda fields, Class. Quantum Grav. 7, no. 4 (1990) L95-L98.

[60] E.A. Zabolotskaya and R.V. Khokhlov, Quasi-plane waves in the nonlinear acoustics of confined beams, Sov. Phys. Acoust. 15 (1969) 35-40.

[61] I. Zakharevich, Nonlinear wave equation, nonlinear Riemann problem, and the twistor transform of Veronese webs, arXiv:math-ph/0006001.

[62] E.V. Zakharov, Dispersionless limit of integrable systems in $2+1$ dimensions, in: Singular Limits of Dispersive Waves, Ed. N.M. Ercolani et al., Plenum Press, NY (1994) 165-174. 